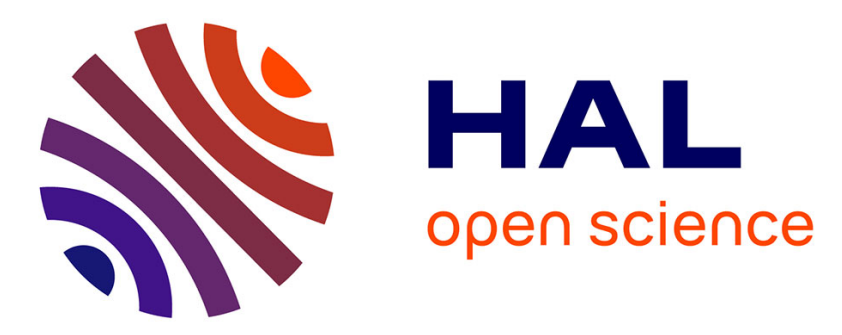

\title{
Transitional regime of electron resonant interaction with whistler-mode waves in inhomogeneous space plasma
}

\author{
A. Artemyev, A. Neishtadt, A. Vasiliev, D. Mourenas
}

\section{To cite this version:}

A. Artemyev, A. Neishtadt, A. Vasiliev, D. Mourenas. Transitional regime of electron resonant interaction with whistler-mode waves in inhomogeneous space plasma. Physical Review E , 2021, 104 (5), 10.1103/PhysRevE.104.055203 . insu-03467046

\section{HAL Id: insu-03467046 \\ https://hal-insu.archives-ouvertes.fr/insu-03467046}

Submitted on 6 Dec 2021

HAL is a multi-disciplinary open access archive for the deposit and dissemination of scientific research documents, whether they are published or not. The documents may come from teaching and research institutions in France or abroad, or from public or private research centers.
L'archive ouverte pluridisciplinaire HAL, est destinée au dépôt et à la diffusion de documents scientifiques de niveau recherche, publiés ou non, émanant des établissements d'enseignement et de recherche français ou étrangers, des laboratoires publics ou privés.

$$
\text { Copyright }
$$




\title{
Transitional regime of electron resonant interaction with whistler-mode waves in inhomogeneous space plasma
}

\author{
A. V. Artemyev,,$^{1,2, *}$ A. I. Neishtadt $\odot,{ }^{2,3}$ A. A. Vasiliev $\odot,{ }^{2}$ and D. Mourenas $\odot^{4}$ \\ ${ }^{1}$ Institute of Geophysics and Planetary Physics, University of California, Los Angeles, California, USA \\ ${ }^{2}$ Space Research Institute RAS, Moscow, Russia \\ ${ }^{3}$ Department of Mathematical Sciences, Loughborough University, Loughborough LE11 3TU, United Kingdom \\ ${ }^{4}$ LPC2E, CNRS, 3 Av. de la Recherche Scientifique, Orléans, France
}

(Received 28 July 2021; accepted 21 October 2021; published 11 November 2021)

\begin{abstract}
Resonances with electromagnetic whistler-mode waves are the primary driver for the formation and dynamics of energetic electron fluxes in various space plasma systems, including shock waves and planetary radiation belts. The basic and most elaborated theoretical framework for the description of the integral effect of multiple resonant interactions is the quasilinear theory, which operates through electron diffusion in velocity space. The quasilinear diffusion rate scales linearly with the wave intensity, $D_{\mathrm{QL}} \sim B_{w}^{2}$, which should be small enough to satisfy the applicability criteria of this theory. Spacecraft measurements, however, often detect whistle-mode waves sufficiently intense to resonate with electrons nonlinearly. Such nonlinear resonant interactions imply effects of phase trapping and phase bunching, which may quickly change the electron fluxes in a nondiffusive manner. Both regimes of electron resonant interactions (diffusive and nonlinear) are well studied, but there is no theory quantifying the transition between these two regimes. In this paper we describe the integral effect of nonlinear electron interactions with whistler-mode waves in terms of the timescale of electron distribution relaxation, $\sim 1 / D_{\mathrm{NL}}$. We determine the scaling of $D_{\mathrm{NL}}$ with wave intensity $B_{w}^{2}$ and other main wave characteristics, such as wave-packet size. The comparison of $D_{\mathrm{QL}}$ and $D_{\mathrm{NL}}$ provides the range of wave intensity and wave-packet sizes where the electron distribution evolves at the same rates for the diffusive and nonlinear resonant regimes. The obtained results are discussed in the context of energetic electron dynamics in the Earth's radiation belt.
\end{abstract}

DOI: 10.1103/PhysRevE.104.055203

\section{INTRODUCTION}

Wave-particle resonant interactions in space plasma play a crucial role in momentum and energy exchange between different charged particle populations in absence of particle collisions. Two main regimes of such interactions are quasilinear diffusion $[1,2]$ and nonlinear resonant interactions $[3,4]$. The most advanced theoretical approaches for these two regimes $[5,6]$ have been developed to model the dynamics of energetic electron populations in the Earth magnetosphere, where electrons interact resonantly with whistler-mode waves. Electron interactions with low-amplitude broadband whistlermode waves are well described by the diffusion model proposed in Ref. [7], whereas the model of resonant interactions with intense coherent waves describes such fast nondiffusive effects as phase trapping [8,9]. Many aspects of these two models have been confirmed and verified with spacecraft observations of long-term (diffusive) electron flux evolution (e.g., Refs. [10,11]) or rapid (nondiffusive) electron acceleration (e.g., Refs. [12-14]) and losses (e.g., Ref. [15]). The same two model approaches (quasilinear diffusion and nonlinear resonances) can describe various wave-particle interactions in the solar wind (e.g., Refs. [16-18]), planetary and

*aartemyev@igpp.ucla.edu interplanetary shock waves (e.g., Refs. $[19,20]$ ), and aurora acceleration region (e.g., Refs. [21,22]).

The basic equations for the quasilinear diffusion model have been initially derived for a homogeneous plasma [5,23], and then generalized for inhomogeneous magnetic traps, like the Earth dipole field [24,25]. An interesting and important aspect of such a generalization is the significant relaxation of the broad wave spectrum requirement (see Refs. $[6,26]$ ). Indeed, for coherent waves the resonance width, a crucial parameter for the diffusive model, can be determined by the magnetic field inhomogeneity [27-29] and/or the wave frequency drift [30-32]. Electron resonant interactions with coherent whistler-mode waves in the Earth's magnetosphere can be described by a quasilinear diffusion model [33] if wave intensity is sufficiently small. Therefore, both regimes of wave-particle interaction (quasilinear diffusion and nonlinear resonances) can operate for the same coherent monochromatic wave, and only wave intensity determines the relevant regime. Thus, the question arises of the transition between these two regimes (see, e.g., discussion in Refs. [34-36]).

The probabilistic distribution of whistler-mode wave intensities in the Earth's magnetosphere contains a large population of low-intensity waves (presumably interacting with electrons diffusively), but this distribution is not Gaussian and there is a significant population of whistler-mode waves sufficiently intense to resonate with electrons nonlinearly [37-39]. The quasilinear diffusion model relies on the average wave 
intensity presumably dominated by low-intensity waves, whereas the nonlinear interaction model invokes the much more rarely observed intense waves. How do such rare nonlinear resonances alter the generally diffusive electron flux evolution? And can the contribution of intense waves be described by a simple increase of diffusion rates of the quasilinear model? To address these questions, we should describe the transition between these two regimes of wave-particle interactions. This topic is the focus of our study. We start with basic equations of wave-particle resonant interactions for the most widespread case of field-aligned whistler-mode waves. Then we derive the main characteristics of resonant electron dynamics for both regimes of resonant interactions. We use the mapping technique for such interactions [40,41] to derive the main scaling laws of the transition between quasilinear and nonlinear interactions. Then we discuss the obtained results and summarize them.

\section{FIELD-ALIGNED WHISTLER-MODE WAVES}

We start with the Hamiltonian of a relativistic electron [the charge is $-e$, the rest mass is $m_{e}$, and energy is $E=$ $m_{e} c^{2}(\gamma-1)$, where $c$ is the speed of light and $\gamma$ is the Lorentz factor] moving in the inhomogeneous background magnetic field $B_{0}(s)$ (where $s$ is the field-aligned coordinate) and whistler-mode wave field [29,42]:

$$
\begin{aligned}
& H=m_{e} c^{2} \gamma+\sqrt{\frac{2 I_{x} \Omega_{0}}{m_{e} c^{2}}} \frac{e B_{w}}{k \gamma} \sin (\phi+\psi) \\
& \gamma=\sqrt{1+\left(\frac{p_{\|}}{m_{e} c}\right)^{2}+\frac{2 I_{x} \Omega_{0}}{m_{e} c^{2}}},
\end{aligned}
$$

where $p_{\|}$is the field-aligned momentum (conjugate to $s$ ); $\Omega_{0}=e B_{0}(s) / m_{e} c$ is the electron gyrofrequency; $I_{x}$ and $\psi$ are magnetic moment (normalized in such a way that $2 I_{x} \Omega_{0}$ is the perpendicular energy) and the conjugate gyrophase; $B_{w}(s)$ is the wave magnetic field amplitude; $\phi$ is the wave phase, with $\partial \phi / \partial s=k(s)$ the wave number; and $-\partial \phi / \partial t=\omega=$ const the wave frequency. We use the cold plasma dispersion relation for $k c / \omega_{\mathrm{pe}}=\left[\Omega_{0}(s) / \omega-1\right]^{-1 / 2}$ with a constant plasma frequency $\omega_{\text {pe }}$. The spatial scale of the background magnetic field inhomogeneity $R$ is much larger than the wavelength $(R k \gg 1)$, whereas the wave amplitude is much smaller than the background magnetic field $\left(B_{w} / B_{0} \ll 1\right)$.

Hamiltonian equations for (1) are

$$
\begin{aligned}
\dot{p}_{\|} & =-\frac{I_{x}}{\gamma} \frac{\partial \Omega_{0}}{\partial s}-\sqrt{\frac{2 I_{x} \Omega_{0}}{m_{e} c^{2}}} \frac{e B_{w}}{\gamma} \cos (\phi+\psi), \\
\dot{s} & =\frac{p_{\|}}{m_{e} \gamma}, \quad \dot{\psi}=\frac{\Omega_{0}}{\gamma}+\sqrt{\frac{2 I_{x} \Omega_{0}}{m_{e} c^{2}}} \frac{e B_{w}}{2 I_{x} k \gamma} \sin (\phi+\psi), \\
\dot{I}_{x} & =-\sqrt{\frac{2 I_{x} \Omega_{0}}{m_{e} c^{2}}} \frac{e B_{w}}{k \gamma} \cos (\phi+\psi),
\end{aligned}
$$

where the rates of variation of $I_{x}$ and $p_{\|}$are much smaller than the rate of variation of $\psi$, because $\Omega_{0} \gg c / R$ and $B_{0} \gg B_{w}$. Therefore, $p_{\|}, s$, and $I_{x}$ are slow variables, and both the wave phase $\phi$ and gyrophase $\psi$ are fast variables.
In the absence of a wave $\left(B_{w}=0\right)$ the electron moves with constant energy $\gamma$ and magnetic moment $I_{x}$ [because the Hamiltonian does not depend on $\psi$ and wave phase $\phi(s, t)]$. Therefore, each trajectory can be characterized by a pair of initial $\left(\gamma, I_{x}\right)$. Instead of $I_{x}$, it is often more convenient to use the electron pitch angle $\alpha_{0}$ determined at the $B_{0}$ minimum (at the equator, $s=0$ ): $2 I_{x} \Omega_{0}(0) / m_{e} c^{2}=\left(\gamma^{2}-1\right) \sin ^{2} \alpha_{0}$. The classical magnetic field configuration of the Earth magnetosphere includes $B_{0}(s)$ growing away from the equator $s=0$ [e.g., the dipole field is $B_{0}=B_{0}(0) \sqrt{1+3 \sin ^{2} \lambda} / \cos ^{6} \lambda$ with magnetic latitude $\lambda$ defined as $\left.d s / R=\sqrt{1+3 \sin ^{2} \lambda} \cos \lambda d \lambda\right]$. In such a field, electrons are moving within a magnetic trap: $\quad p_{\|}= \pm \sqrt{1-\gamma^{2}-2 I_{x} \Omega_{0}(s) / m_{e} c^{2}} \quad$ is oscillating between $\pm \sqrt{1-\gamma^{2}-2 I_{x} \Omega_{0}(0) / m_{e} c^{2}}$ values and reaches zeros at the mirror coordinates $s_{\max \text { min }}$ defined as $\Omega_{0}\left(s_{\max , \min }\right) \sin ^{2} \alpha_{0} / \Omega_{0}(0)=1$.

In the presence of a wave $\left(B_{w} \neq 0\right)$, the first-order cyclotron resonance condition $\dot{\phi}+\dot{\psi}=k p_{z} / m_{e} \gamma-\omega+$ $\Omega_{0} / \gamma=0$ determines the position of resonance $s_{R}$ for given electron energy and pitch angle $\alpha_{0}$ with the resonant momentum $p_{z}=-\sqrt{1-\gamma^{2}-\Omega_{0}\left(s_{R}\right) \sin ^{2} \alpha_{0} / \Omega_{0}(0)}$.

The equation for $p_{\|}$in system (2) contains two terms: the mirror force and the wave force. For the diffusive regime of resonant interactions the mirror force is much larger than the wave force, and thus wave effects can be calculated using the integration of wave field over unperturbed electron trajectory. For nonlinear resonant interactions, these two terms should be of the same order, i.e., the wave force should be able to compete with the mirror force and change the electron trajectory substantially. The ratio of the magnitudes of these two forces is $\left(B_{w} / B_{0}\right) R \Omega_{0} / c$. Thus, the regime of nonlinear resonant interaction requires $B_{w} / B_{0} \sim c / R \Omega_{0}$, whereas if $B_{w} / B_{0} \ll c / R \Omega_{0}$, then the resonant interaction should be diffusive. Figure 1 shows electron trajectories for these two regimes: Figure 1(a) shows the energy scattering with a mean zero for $B_{w} / B_{0} \ll c / R \Omega_{0}$, whereas Fig. 1(b) shows a significant energy change for $B_{w} / B_{0} \sim c / R \Omega$. The nonlinear regime is characterized by two processes: A small number of particles experience phase trapping with a large energy increase, whereas most of the particles experience nonlinear scattering (phase bunching) with a small energy decrease. The long-term dynamics (multiple resonances) for a single electron would resemble the random fluctuations of a diffusive regime [see Fig. 1(c)] with some sort of cycle for the nonlinear regime [see Fig. 1(d)]. This cycle consists in multiple drifts to smaller energy due to the nonlinear scattering and rare trappings with energy increase. We aim to describe the transition between these two regimes for an ensemble of electrons. For this reason we shall start with the quantification of the main characteristics of electron dynamics in both regimes.

\section{EQUATIONS OF PARTICLE MOTION AROUND THE RESONANCE}

The diffusive regime of resonant interaction can be described by the diffusion coefficient $D_{\mathrm{QL}}$, whereas the nonlinear regime is characterized by energy changes due to the nonlinear scattering $\Delta \gamma_{\text {scat }}$, energy changes due to trapping 

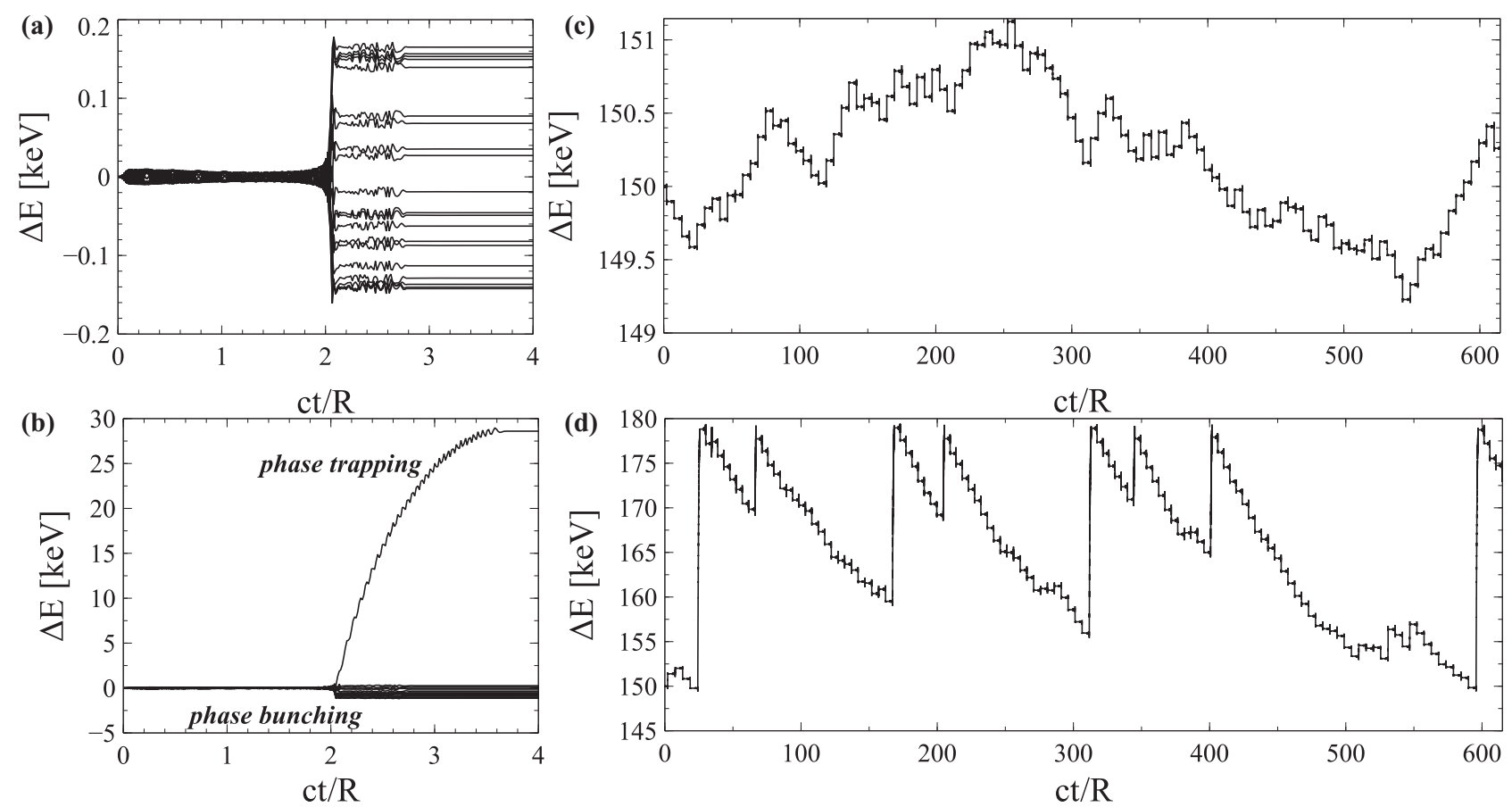

FIG. 1. Trajectories of electrons resonantly interacting with a parallel whistler-mode wave (one resonant interaction is shown): Wave amplitude is sufficiently low and electrons are diffusively scattered (a) and wave amplitude is high and electrons experience phase bunching and phase trapping (b). Long-term dynamics of electron trajectories ( $\sim 100$ resonances) for low (c) and high (d) wave amplitudes. All electrons have the same initial energy of $150 \mathrm{keV}$ and pitch angle $45^{\circ}$. The background magnetic field is the Earth's dipole field with an equatorial magnitude corresponding to 6 Earth radii away from the planet (the outer radiation belt). Wave characteristics are typical for parallel whistler-mode waves: $\omega / \Omega_{0}(0)=0.25, B_{w}=20 \mathrm{pT}$ for (a) and (c) and $B_{w}=200 \mathrm{pT}$ for (b) and (d) $[43,44]$. The background plasma density is given by an empirical model [45].

$\Delta \gamma_{\text {trap }}$, and the probability $\Pi$ of trapping [46-48]. In this section we demonstrate the relations between these different characteristics. First, we should consider Hamiltonian (1) around the resonance. We follow the procedure described in Refs. $[49,50]$ and introduce the new phase $\zeta=\phi+\psi$ through the generating function $W=(\phi+\psi) I+P_{\|} s$. In the new variables, the Hamiltonian takes the form:

$$
\begin{gathered}
H_{I}=m_{e} c^{2} \gamma-\omega I+\sqrt{\frac{2 I \Omega_{0}}{m_{e} c^{2}}} \frac{e B_{w}}{k \gamma} \sin \zeta \\
\gamma=\sqrt{1+\left(\frac{P_{\|}+k I}{m_{e} c}\right)^{2}+\frac{2 I \Omega_{0}}{m_{e} c^{2}}},
\end{gathered}
$$

where we keep the same notation for $s$ conjugate to $P_{\|}=$ $p_{\|}-k I$ and take into account that new Hamiltonian $H_{I}=$ $H+\partial W / \partial t$. New momentum $I$ conjugate to $\zeta$ equals $I_{x}$.

Although the most intense whistler-mode waves in the Earth's radiation belts are chorus waves with $\partial \omega / \partial t \neq 0$ [51-53], for simplicity of the system description we consider here $\omega=$ const. This assumption excludes several potentially important effects of nonlinear resonant interactions [30,54] but allows a reduction of the system dimension. For $\omega=$ const Hamiltonian (3) does not depend on time, and thus $\gamma-\omega I / m_{e} c^{2}=h$ is the integral of motion. This relation between energy and $I$ for a given frequency $\omega$ makes the resonant interaction effectively $1 \mathrm{D}$, i.e., any electron energy and pitch-angle change obeys $\gamma-\left[\omega / 2 \Omega_{0}(0)\right]\left(\gamma^{2}-1\right) \sin ^{2} \alpha_{0}=$ $h=$ const. Figures 2(a) and 2(b) shows such 1D motion in the $\left(\gamma, \alpha_{0}\right)$ plane for diffusive and nonlinear resonant interactions of electrons from Figs. 1(c) and 1(d). Note although the classical quasilinear theory was developed for a broadband wave spectrum (i.e., for $\omega \neq$ const) [1,2], in the inhomogeneous plasma (with $\partial \Omega_{0} / \partial s \neq 0$ ) this theory can be generalized for a narrow-band wave $[27,33]$. Therefore, we may compare diffusive scattering of electrons by a coherent whistler-mode wave with $\omega=$ const and compare efficiency of such diffusive scattering with the nonlinear resonant effects.

Therefore, we can introduce the 1D electron distribution $f(\gamma)$ for $h=$ const and follow the evolution of this distribution in these two different regimes. Figure 2(c) shows that the initially localized $f(\gamma)$ is diffusively spread for low wave intensity. Phase trapping and nonlinear scattering modify the initially localized $f(\gamma)$ distribution in a nondiffusive manner: There is a drift of the entire distribution due to nonlinear scattering and the appearance of a high-energy electron population due to trappings [see Fig. 2(d)]. An interesting and important property of the wave-particle resonant interaction is that both regimes (diffusion and nonlinear resonances) ultimately result in $f(\gamma)$ relaxation to a plateau with $\partial f /\left.\partial \gamma\right|_{h=\text { const }} \rightarrow 0$ (see Ref. [55]). The energy ranges are different for the two regimes, and the diffusive relaxation would work over a wider energy range because $B_{w} / B_{0} \sim$ $c / R \Omega_{0}$ can be satisfied only in a certain range of magnetic latitudes corresponding to a certain range of resonant energies $\gamma \in\left[\gamma_{\max }, \gamma_{\min }\right]$, where $\gamma_{\max }$ min are determined by $B_{0}(s)$ and $B_{w}(s)$ profiles. On the other hand, due to a resonance broadening effect (i.e., the finite width of the resonance in 

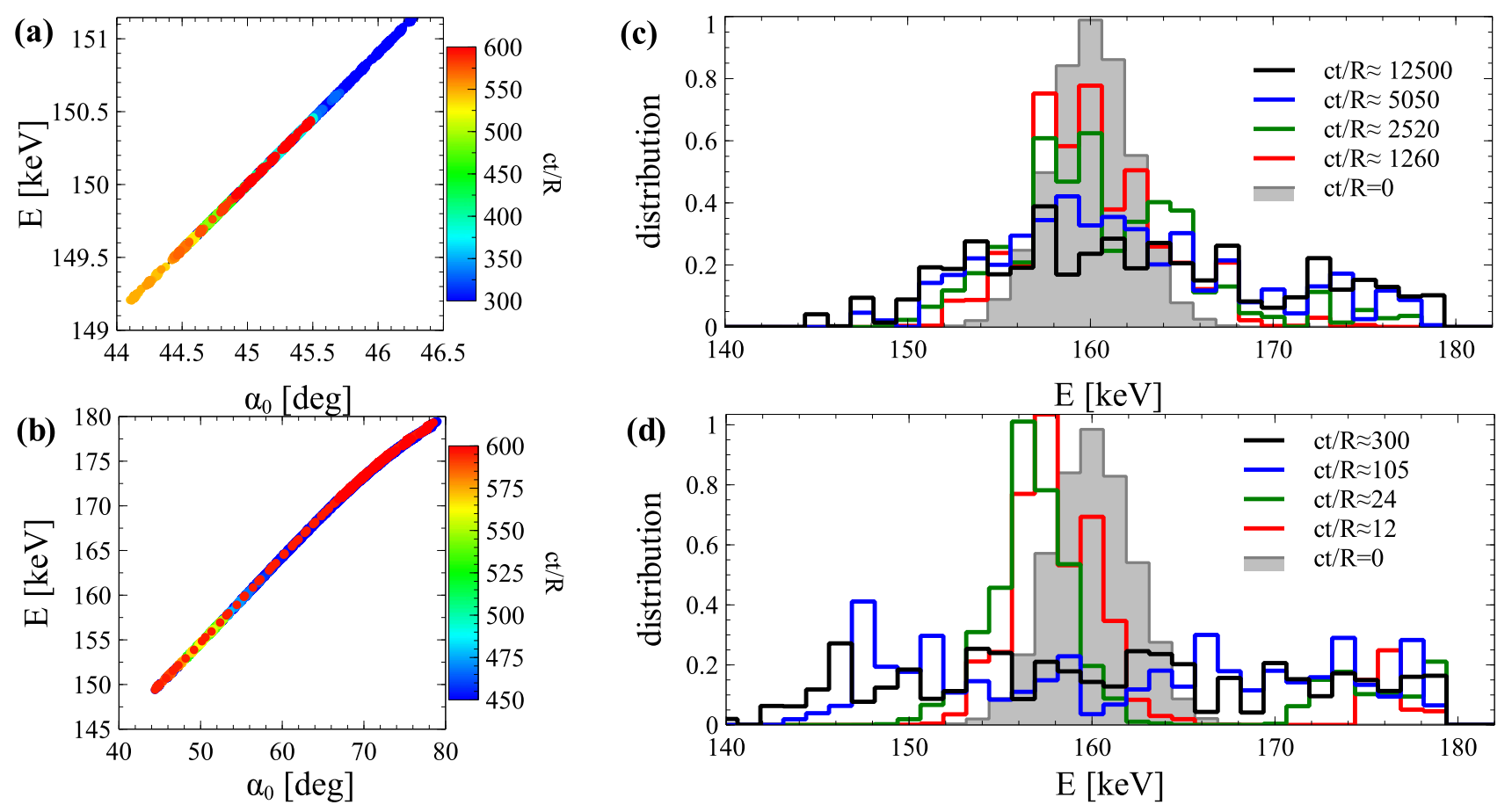

FIG. 2. Two trajectories from Figs. 1(c) and 1(d) are shown in the (energy, pitch angle) plane for diffusive (a) and nonlinear (b) resonant interactions (color shows time). Evolution of an initially localized peak of distribution $f(\gamma)$ for the same $h=\gamma-\left[\omega / 2 \Omega_{0}(0)\right]\left(\gamma^{2}-1\right) \sin ^{2} \alpha_{0}$ as from panels (a) and (b): diffusive (c) and nonlinear (d) resonant interactions. Note that timescales are different for (c) and (d) panels.

the velocity space $\sim \sqrt{B_{w} / B_{0}}$, see Refs. [9,56]) the nonlinear resonant interaction may affect energies formally nonresonant in the diffusive regime (e.g., [57,58]. The timescales of $f(\gamma)$ relaxation are also different for the two regimes: The diffusive relaxation requires more time and Fig. 2(d) shows how $f(\gamma)$ continues spreading outside the energy range of nonlinear resonances where the plateau forms first.

The resonance condition for Hamiltonian (3) is given by equation $\dot{\zeta}=0$ and this equation determines the resonant momentum $I_{R}$ :

$$
\begin{aligned}
\frac{k I_{R}}{m_{e} c}= & -\frac{P_{\|}}{m_{e} c}-\frac{\Omega_{0}}{k c}+\left[\left(\frac{k c}{\omega}\right)^{2}-1\right]^{-1 / 2} \\
& \times\left[1-\left(\frac{\Omega_{0}}{k c}\right)^{2}-2 \frac{\Omega_{0}}{k c} \frac{P_{\|}}{m_{e} c}\right]^{1 / 2} .
\end{aligned}
$$

We substitute $I_{R}$ into the $\gamma$ expression and obtain the resonant energy

$$
\gamma_{R}=\frac{k c / \omega}{\sqrt{(k c / \omega)^{2}-1}}\left[1-\left(\frac{\Omega_{0}}{k c}\right)^{2}-2 \frac{\Omega_{0}}{k c} \frac{P_{\|}}{m_{e} c}\right]^{1 / 2} .
$$

Substituting $P_{\|}$from $h=\gamma_{R}-\omega I_{R} / m_{e} c^{2}$ into Eq. (5) we can obtain $\gamma_{R}(s)$. This function determines the resonant energy $\gamma_{R}$ at each value of $s$ coordinate. Therefore, we can use $\gamma_{R}$ instead of $s$ (instead of resonant $s_{R}$ ) and for $h=$ const we can rewrite $s$ profiles of all system parameters (wave amplitude, background magnetic field) as $\gamma_{R}$ profiles.
To determine the electron dynamics (energy change) around the resonance, we expand Hamiltonian (3) as

$$
\begin{gathered}
H_{I}=m_{e} c^{2} \gamma_{R}-\omega I_{R}+\frac{1}{2} m_{e} c^{2} g\left(I-I_{R}\right)^{2} \\
+\sqrt{\frac{2 I_{R} \Omega_{0}}{m_{e} c^{2}}} \frac{e B_{w}}{k \gamma_{R}} \sin \zeta \\
g= \\
\left.\frac{\partial^{2} \gamma}{\partial I^{2}}\right|_{I=I_{R}}=\frac{k^{2}}{m_{e}^{2} c^{2}} .
\end{gathered}
$$

Such expansion can be done for $2 \Omega_{0} I / m_{e} c^{2} \gg B_{w} / B_{0}$, whereas for smaller $I$ an alternative consideration would be required (see Refs. [59,60]).

We use the generating function $Q=\left(I-I_{R}\right) \zeta+P_{\|} S$ to introduce $P_{\zeta}=I-I_{R}$. The new Hamiltonian consists of two parts $\Lambda=m_{e} c^{2} \gamma_{R}-\omega I_{R}$ and $H_{\zeta}$ :

$$
H_{\zeta}=\frac{1}{2} m_{e} c^{2} g P_{\zeta}^{2}+\left\{\Lambda, I_{R}\right\} \zeta+\sqrt{\frac{2 I_{R} \Omega_{0}}{m_{e} c^{2}}} \frac{e B_{w}}{k \gamma_{R}} \sin \zeta,
$$

where $\Lambda$ depends on new variables $p=P_{\|}+\zeta \partial I_{R} / \partial s, q=$ $s-\zeta \partial I_{R} / \partial P_{\|}$, and we expand $H_{I}$ over $\zeta \partial I_{R} / \partial s,-\zeta \partial I_{R} / \partial P_{\|}$to get the Poisson brackets $\{\cdot, \cdot\}$ (see, e.g., Refs. [42,61]). Hamiltonian $H_{\zeta}$ describes the electron dynamics on the $\left(\zeta, P_{\zeta}\right)$ plane (around the resonance $P_{\zeta}=0$ ), and coefficients of this Hamiltonian depend on slowly changing $(p, q)$. Note that the dependence on $(p, q)$ can be rewritten as a dependence on $\gamma_{R}$, because $(p, q) \approx\left(P_{\|}, s\right)$ where $s=s_{R}$ and $P_{\|}=P_{\|}\left(s_{R}\right)$ due to $\gamma_{R}-\omega I_{R} / m_{e} c^{2}=h$ conservation.

Hamiltonian (7) describes the classical system of a pendulum with a torque (e.g., Ref. [62]), and the phase portraits of 


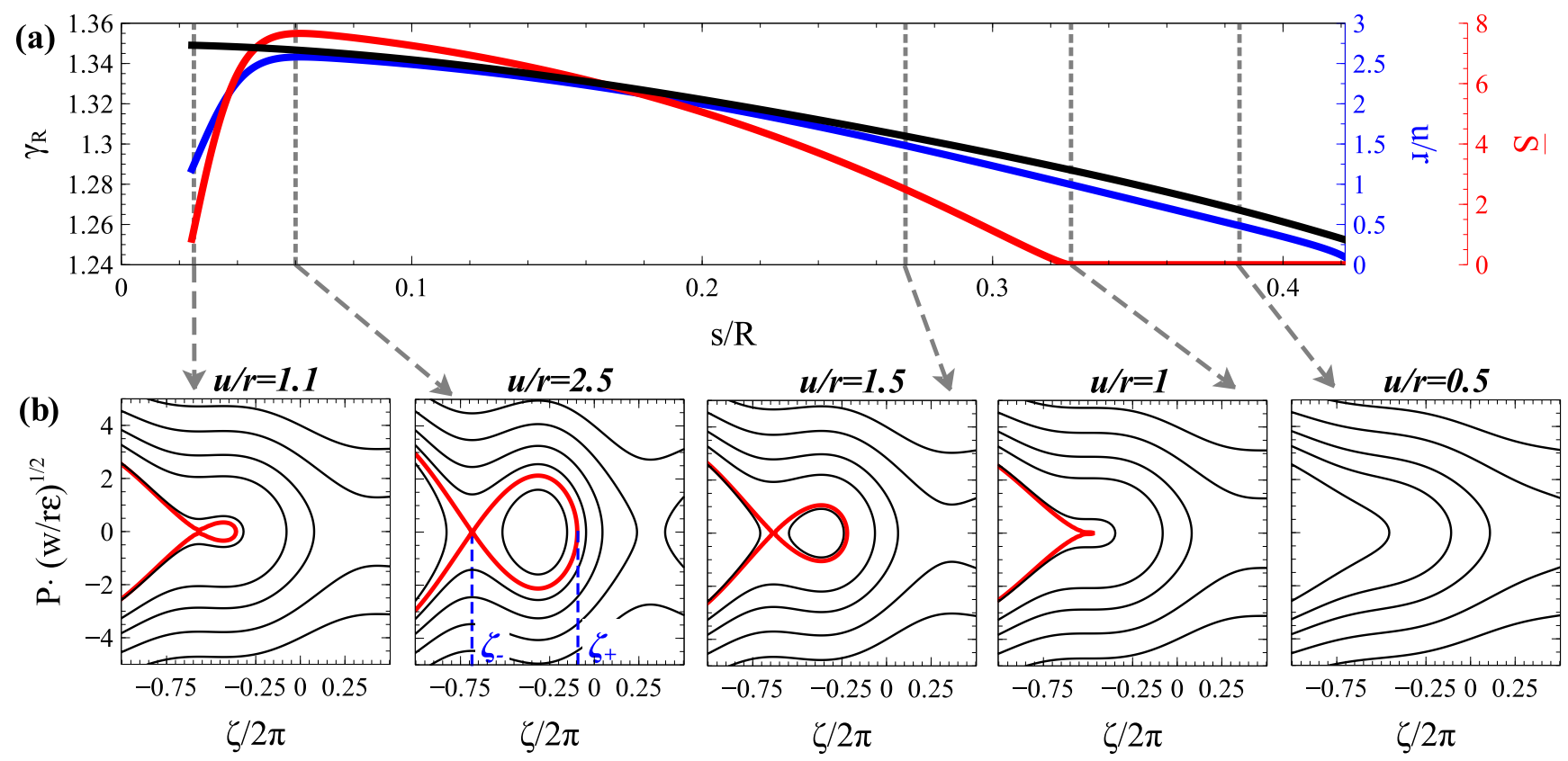

FIG. 3. The profile of $u / r$ ratio (a). Phase portraits of Hamiltonian (8) for different $u / r$ ratios (b). Profiles of $\gamma_{R}$ and $\bar{S}=S\left[R \Omega_{0}(0) / c\right]^{1 / 2}$ along $s$ coordinate [note $R \Omega_{0}(0) / c$ is of the order of $1 / \varepsilon$ for the system parameters] (a). System parameters correspond to the trajectory shown in Fig. 1(b).

this system with different values of coefficients are shown in Fig. 3(a). To describe the main properties of this Hamiltonian, let us rewrite it in a dimensionless form $\mathcal{H}=H / m_{e} c^{2}$ :

$$
\mathcal{H}=\frac{1}{2} w P^{2}+\varepsilon(r \zeta+u \sin \zeta)
$$

where $P=P_{\zeta} / P_{0}, w=g P_{0}^{2}\left(P_{0}\right.$ is the $P_{\zeta}$ magnitude), max $B_{w} / B_{0}(0)=\varepsilon, u=\sqrt{2 I_{R} \Omega_{0} / m_{e} c^{2}}\left[\Omega_{0}(0) / c k \gamma_{R}\right]\left(B_{w} / \max B_{w}\right)$, and $r=\left\{\Lambda, I_{R}\right\} / m_{e} c^{2} \varepsilon$ and time renormalized to $1 / P_{0}$ to keep Hamiltonian form of equations. Note $\left\{\Lambda, I_{R}\right\} \propto c / R \Omega_{0}$ and we assume that $c / R \Omega_{0} \leqslant B_{w} / B_{0}$.

For a typical wave field distribution along magnetic field lines (in statistical satellite observations, $B_{w}$ is generally small close to the equator $s=0$ and at high magnetic latitudes $s \rightarrow 1$, with a $B_{w}$ maximum at medium latitudes) (see Refs. [63,64]), there are three spatial regions with different phase portraits of Hamiltonian (8). The wave is generated near the equator $s=0$ and moves to higher $s$ (i.e., wave number $k>0$ ), whereas the resonant electron moves from high $s$ to the equator because the resonant momentum $p_{\|}=$ $\left(\gamma \omega-\Omega_{0}\right) / k$ is negative for not-too-high $\gamma$. Thus, we consider the phase portrait evolution moving from large $s$ to the equator. At high $s$-wave amplitude is small and $|r|>u$. Thus, the phase portrait totally consists of trajectories crossing the resonance $P=0$ once (open trajectories). As $s$ decreases, we obtain $u>|r|$. The corresponding phase portrait contains closed trajectories and particles on these trajectories are trapped around the resonance (i.e., cross $P=0$ many times). The growth of the area $S$ filled by such trapped trajectories in $(\zeta, P)$ plane implies that particles from the region with open trajectories can be trapped into the resonance. The area $S$ reaches its maximum at a value of $s$ where $|u / r|$ is maximal; as $s$ further decreases, this area starts decreasing. Such area
$S$ decrease corresponds to particles detrapping (escape) from the resonance. Close to the equator $s=0$ all particles will be detrapped and only open trajectories remain in the phase portrait (see Fig. 3). This assumption of $S=0$ at the equator simplifies the model description of the trapped electron dynamics. Although in more realistic simulations with a finite size of the wave generation region [65-70] trapped electrons may cross the equator without detrapping, in general this effect should not significantly alter the trapped electron energy and pitch-angle changes.

The evolution of the area $S$ with closed trajectories along $S$ is much slower than trapped particles rotation around $P=0$, and thus this periodic rotation has an adiabatic invariant $I_{\zeta}=$ $(2 \pi)^{-1} \oint P d \zeta$. At the moment of trapping $I_{\zeta}$ equals $S / 2 \pi$ (and $S$ increases), and due to $I_{\zeta}$ conservation the detrapping should appear when $S$ decreases and comes back to the value $2 \pi I_{\zeta}$. However, during this $S$ evolution the resonant energy would change (see the example of $\gamma_{R}$ profile along $s$ in Fig. 3). Thus, between trapping and detrapping electrons gain energy $\Delta \gamma_{\text {trap }}$, and this energy gain is determined by $S\left(\gamma_{R}\right)$ curve $S=\oint P_{\zeta} d \zeta$ :

$$
S=2 \sqrt{\frac{2 \varepsilon}{w}} \int_{\zeta_{-}}^{\zeta_{+}} \sqrt{\left(r \zeta_{+}+u \sin \zeta_{+}\right)-(r \zeta+u \sin \zeta)} d \zeta
$$

where $\zeta_{ \pm}$are shown in Fig. 3. The amount of particles that will be trapped during a single resonant interaction (i.e., the probability of trapping, $\Pi$ ) is determined by the gradient of $S$ (see Refs. [28,49,71]): $\Pi=\left(\omega / 2 \pi m_{e} c^{2}\right)(\partial S / \partial \gamma)$.

All particles that are not trapped cross the resonance $P=0$ once, moving along open trajectories. These particles are scattered with the energy change $\Delta \gamma=\omega \Delta I / m_{e} c^{2}$, which 
depends on the phase $\zeta_{R}$ at the resonance:

$$
\begin{aligned}
\Delta \gamma & =-\int_{-\infty}^{\zeta R} \frac{2 \sqrt{\frac{2 \omega^{2}}{m_{e} c^{2} g}} \sqrt{\frac{2 I_{R} \Omega_{0}}{m_{e} c^{2}}} \frac{e B_{w}}{k \gamma_{R} m_{e} c^{2}} \cos \zeta}{\sqrt{H_{\zeta}-\left\{\Lambda, I_{R}\right\} \zeta-\sqrt{\frac{2 I_{R} \Omega_{0}}{m_{e} c^{2}}} \frac{e B_{w}}{k \gamma_{R}} \sin \zeta}} d \zeta \\
& =-2 \sqrt{\frac{2 \varepsilon \omega^{2}}{m_{e}^{2} c^{4} g}} \int_{-\infty}^{\zeta_{R}} \frac{u \cos \zeta}{\sqrt{2 \pi \theta r-r \zeta-u \sin \zeta}} d \zeta,
\end{aligned}
$$

where $2 \pi \theta=\zeta_{R}+(u / r) \sin \zeta_{R}(\bmod 2 \pi)$. The function $\Delta \gamma(\theta)$ is periodic; its mean value over $\theta \in[0,1]$ is equal to $\Delta \gamma_{\text {scat }}=\langle\Delta \gamma(\theta)\rangle_{\theta}=-\left(\omega / 2 \pi m_{e} c^{2}\right) S$ (see Refs. [48,72]). Thus, for the phase portrait with $S \neq 0$ the energy change due to scattering is also determined by the $S\left(\gamma_{R}\right)$ profile. This relation has been verified many times with numerical simulations for electron interactions with whistler-mode waves (see Refs. [42,46,61]). For the phase portrait with $S=0$ we get $\langle\Delta \gamma(\theta)\rangle_{\theta}=0$ (i.e., no energy drift due to the resonance), but $\left\langle(\Delta \gamma(\theta))^{2}\right\rangle_{\theta} \neq 0$ and there is an energy diffusion. Equation (10) shows that for $|u / r| \ll 1$ we get

$$
\Delta \gamma \approx-2 \varepsilon \sqrt{\frac{2 \omega^{2}}{m_{e} c^{2} g}} \int_{-\infty}^{\zeta_{R}} \frac{u \cos \zeta d \zeta}{\sqrt{2 \pi \theta-\left\{\Lambda, I_{R}\right\} \zeta / m_{e} c^{2}}}
$$

and $D_{\mathrm{QL}}=\left\langle(\Delta \gamma(\theta))^{2}\right\rangle_{\theta} \sim \varepsilon^{2}$, as it should be for quasilinear diffusion coefficients proportional to the wave intensity (see detailed comparison of $D_{\mathrm{QL}}$ calculated with Eq. (11) and quasilinear diffusion rates in Refs. [6,33]).

As electron energy changes only at resonances and remains constant between resonances, its evolution for any trajectory can be described by the mapping technique (e.g., Ref. [73]). For the nonlinear resonances such a map takes the form (see Ref. [41]):

$$
\gamma_{n+1}=\gamma_{n}+ \begin{cases}\Delta \gamma_{\text {trap }}, & \theta \in[0, \Pi] \\ \Delta \gamma_{\text {scat }}, & \theta \in(\Pi, 1]\end{cases}
$$

where $n$ is the number of resonance crossings (the number of map iterations). Equation (12) shows that new energy (after $n+1$ resonant interaction) $\gamma_{n+1}$ is the sum of energy $\gamma_{n}$ after previous resonance and energy change due to scattering or trapping. Variable $\theta$ is the rescaled effective energy at the resonance [see Eq. (10)], and the variation of $\theta$ between two resonance crossings is determined by the normalized phase gain $\Delta \zeta \sim \varepsilon^{-1} \gg 1$ (see the equations in Appendix of Ref. [41]). This energy $\theta$ can be treated as a random variable with uniform distribution on $[0,1]$ (see discussion of possible limitations of such a consideration in Ref. [74]). Map (12) shows that for fixed $h$ the entire dynamics of electron energy due to nonlinear resonant interactions is described by a single curve $S(\gamma)$ : The energy change due to scattering is $\Delta \gamma_{\text {scat }}=-\left(\omega / 2 \pi m_{e} c^{2}\right) S$, the probability of trapping is $\Pi=\left(\omega / 2 \pi m_{e} c^{2}\right)(d S / d \gamma)$, and energy change due to trapping $\Delta \gamma_{\text {trap }}$ is defined as the difference of $\gamma_{R}$ between two $S(\gamma)$ values [see scheme in Fig. 4(b)].

To verify the mapping technique described by Eq. (12) we calculate $S(\gamma)$ for $h$ from Fig. 1(b) and iterate a large ensemble of trajectories. Figure 4(a) shows $S(\gamma), \Pi(\gamma)$ profiles, several individual trajectories, and dynamics of $f(\gamma)$ relaxation. Note that to transform iterations $n$ into time, one should use $t_{n+1}=$ $t_{n}+\tau_{b}(\gamma)$, where $\tau_{b}$ is the electron bounce period, i.e., the
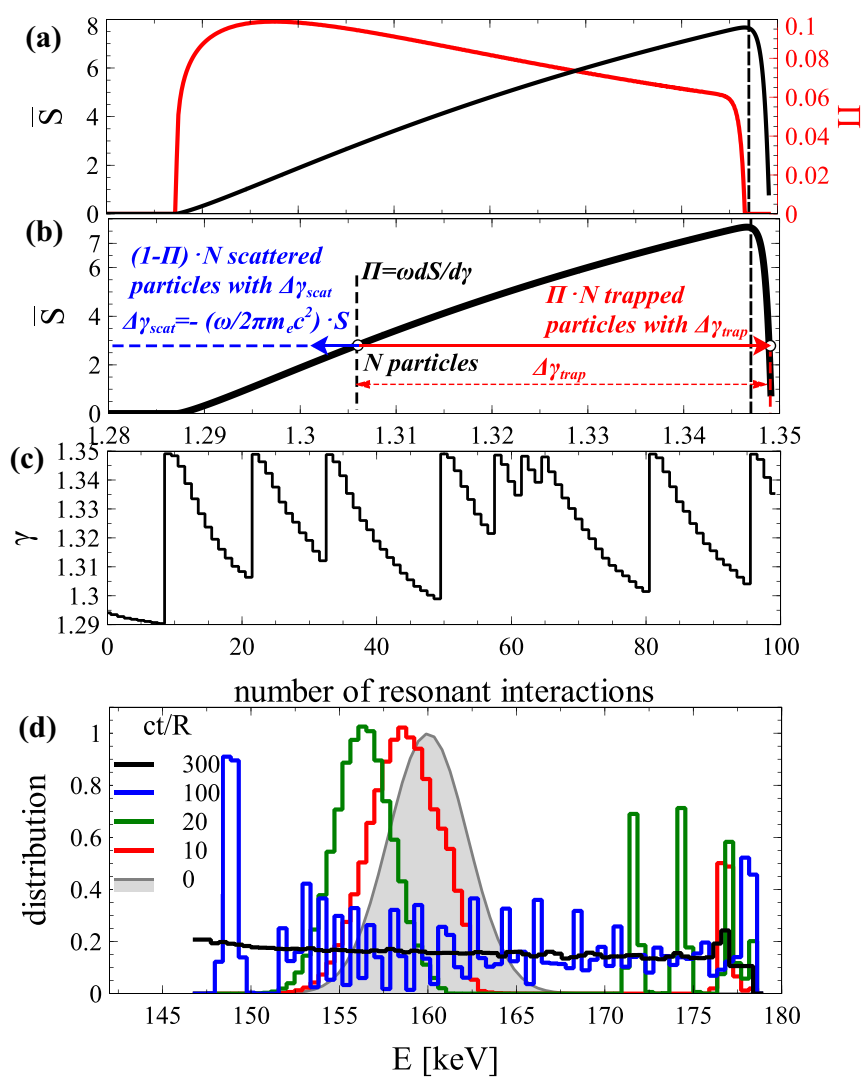

FIG. 4. Panel (a) shows profiles of $S(\gamma), \Pi(\gamma)$ for the trajectory from Fig. 1(b). Schematic view of electron motion along $\gamma$ for given $\bar{S}(\gamma)=S\left(R \Omega_{0}(0) / c\right)^{1 / 2}$ (b) [note $R \Omega_{0}(0) / c$ is of the order of $1 / \varepsilon$ for the system parameters]. An example of trajectory $\gamma(n)$ for $n=0 . .100$ resonant iterations (c). The relaxation of the distribution function $f(\gamma)(\mathrm{d})$. Plots in panels (c) and (d) are obtained with $\operatorname{map}(12)$.

time interval between two resonant interactions (we consider waves only in the $s>0$ hemisphere): For the dipole field $\tau_{b} \approx$ $4\left(1-\gamma^{-2}\right)^{-1 / 2}\left(1.3802-0.6397 \sin ^{3 / 4} \alpha_{0}\right)$ [75]. Comparison of Figs. 2 and 4 demonstrates the applicability of the mapping technique for the description of electron resonant dynamics (see more examples with this approach in Refs. $[41,76]$ ).

To summarize, the nonlinear resonances provide trapping and nonlinear scattering with energy changes described by $S(\gamma)$ curve. In this regime of resonant interaction $S \sim \sqrt{\varepsilon}$ [see Eq. (9)]. As $|u / r|$ decreases, the magnitude of $S$ tends to zero, and for sufficiently small $|u / r|$ there is only diffusion of electrons with the diffusion rate $D_{\mathrm{QL}}=\left\langle(\Delta \gamma)^{2}\right\rangle \sim \varepsilon^{2}$ (see Eq. (11) and Ref. [33]). We aim to investigate the transition between these two regimes and to obtain estimates of the corresponding different timescales of $f(\gamma)$ relaxation.

\section{MAIN SYSTEM SCALINGS}

To consider the transition between nonlinear resonant interaction and quasilinear diffusion, we need to consider a $S \rightarrow 0$ limit for a fixed $\varepsilon$. We start with the evaluation of Hamiltonian (8) with small $S$. Next, we investigate timescales of $f(\gamma)$ relaxation for $S \rightarrow 0$. Then we explain the relation of these timescales and the timescale of electron acceleration 

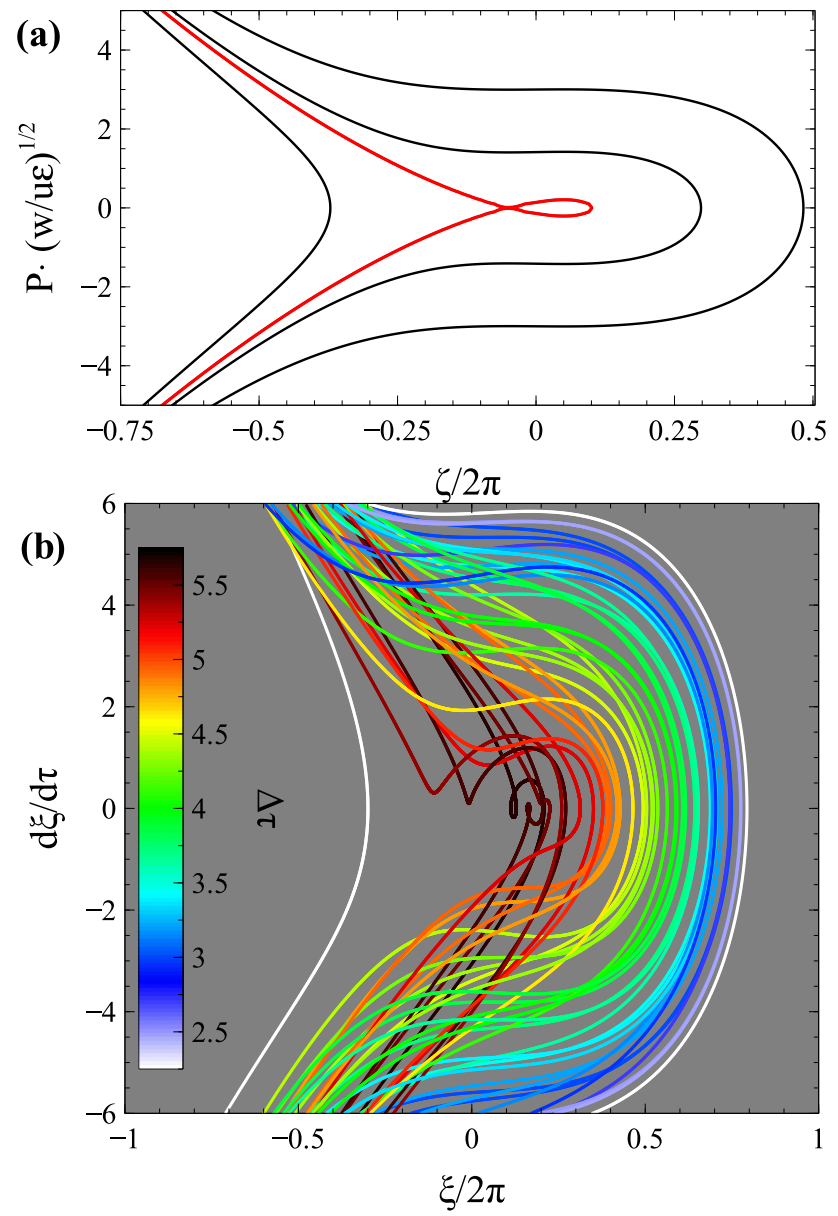

FIG. 5. The phase portrait of Hamiltonian (13) for $r / u$ around 1 (a). Set of trajectories obtained by numerical integration of Eq. (17) with $\delta_{0}=\varepsilon^{2 / 3}$. Color codes the time interval required to cross the shown $d \xi / d \tau$ range.

within the trapping. Finally, all obtained equations are combined together to provide the relation between timescales of $f(\gamma)$ relaxation due to nonlinear resonances and quasilinear diffusion.

\section{A. Threshold $S$ values}

Let us consider Hamiltonian (8) for $|r / u|$ around one, i.e., when $|r|$ becomes smaller than $u$ (condition required for existence of $S \neq 0$ ) only within a short $\gamma_{R}$ range. Then the $\zeta$ range of the area filled with closed trajectories $\left[\zeta \in\left[\zeta_{-}, \zeta_{+}\right]\right.$ in Fig. 3(b)] is small and Hamiltonian (8) can be expanded as:

$$
\begin{aligned}
\mathcal{H} & =\frac{1}{2} w P^{2}+\varepsilon u\left(\frac{r}{u} \zeta+\sin \zeta\right) \\
& \approx \frac{1}{2} w P^{2}+\varepsilon u\left[\left(\frac{r}{u}-1\right) \zeta+\frac{1}{6} \zeta^{3}\right],
\end{aligned}
$$

where $r / u$ slowly changes along the trajectory from $>1$ (no closed trajectories in the phase portrait) to $\min r / u<1$ (maximum area filled by closed trajectories) and then to $>1$ again. Figure 5(a) shows the phase portrait of Hamiltonian (13) for such $r / u<1$. To model this evolution we can write $r / u=$ $1-\delta_{0}+(\varepsilon t)^{2}$ where the small parameter $\delta_{0}>0$ determines how far $|r / u|$ is from 1 and $(\varepsilon t)^{2}$ models the slow evolution of $r / u$ along the trajectory (i.e., we change the slow coordinate $s$ to a slow time here):

$$
\mathcal{H} \approx \frac{1}{2} w P^{2}-\varepsilon u\left\{\left[\delta_{0}-(\varepsilon t)^{2}\right] \zeta-\frac{1}{6} \zeta^{3}\right\}
$$

and $w, u$ can be considered as constant along a short interval of $\varepsilon t \in\left[-\sqrt{\delta_{0}}, \sqrt{\delta_{0}}\right]$. The equation for $S$ for Hamiltonian (14) can be written as

$$
\begin{aligned}
S= & \sqrt{\frac{8 u \varepsilon}{w}} \int_{\zeta_{+}}^{\zeta-}\left\{\left[\delta_{0}-(\varepsilon t)^{2}\right]\left(\zeta-\zeta_{+}\right)-\frac{\zeta^{3}-\zeta_{+}^{3}}{6}\right\}^{1 / 2} d \zeta \\
= & \sqrt{\frac{8 u \varepsilon}{w}} \delta_{0}^{5 / 4}\left(1-\frac{t^{2}}{t_{0}^{2}}\right)^{5 / 4} \\
& \times \int_{\bar{\zeta}_{+}}^{\bar{\zeta}-}\left[\left(\bar{\zeta}-\bar{\zeta}_{+}\right)-\frac{\bar{\zeta}^{3}-\bar{\zeta}_{+}^{3}}{6}\right]^{1 / 2} d \bar{\zeta} \\
= & \sqrt{\frac{8 u \varepsilon}{w}} \delta_{0}^{5 / 4}\left[1-\left(t / t_{0}\right)^{2}\right]^{5 / 4} \frac{(12) 2^{3 / 4}}{5},
\end{aligned}
$$

where $\bar{\zeta}_{+}=-\sqrt{2}, \bar{\zeta}_{-}=\sqrt{8}, t_{0}=\sqrt{\delta_{0}} / \varepsilon$ and $t / t_{0}$ is equivalent to $\gamma_{R}$, the energy at resonance, because different slow time values imply here different values of slow $s$, i.e., different values of resonant $s_{R}$ related to $\gamma_{R}$ through Eq. (5) and $h=$ const. Equation (15) demonstrates that the $\delta_{0}$ parameter controls the magnitude of $S$, and effects of nonlinear interactions should disappear as $\delta_{0} \rightarrow 0$. In the nonlinear regime, there are well separated populations of trapped particles (a small number of particles gaining a large energy) and nonlinearly scattered particles (a large number of particles losing energy). In contrast, in the diffusive regime the numbers of particles gaining and losing energy are (approximately) equal. Therefore, there is a threshold $\delta_{0}$ value (or $S$ value) such that for $\delta_{0}$ below this threshold, we cannot separate trapping and nonlinear scattering. Let us obtain this threshold $\delta_{0}$ (or $S$ ) value.

Hamiltonian equations for Hamiltonian (14) can be combined to get the second-order equation for $\zeta$ :

$$
\frac{d^{2} \zeta}{d \tilde{t}^{2}}=\varepsilon\left[\delta_{0}-(\varepsilon \tilde{t})^{2}-\frac{1}{2} \zeta^{2}\right],
$$

where $\tilde{t}=t \sqrt{u w}$ and $u, w$ are constants of the order of one (not dependent on $\varepsilon$ ). Equation (16) can be rewritten in normalized variables $\tau=\tilde{t} \varepsilon^{1 / 2} \delta_{0}^{1 / 4}, \xi=\zeta / \sqrt{\delta_{0}}$,

$$
\frac{d^{2} \xi}{d \tau^{2}}=\left(1-\frac{\varepsilon}{\delta_{0}^{3 / 2}} \tau^{2}\right)-\frac{1}{2} \xi^{2} .
$$

Equation (17) shows that for $\delta_{0}=\varepsilon^{2 / 3}$ there is no separation of timescales, i.e., the equation describing the electron motion around the resonance does not contain a slow time. Thus, both trapped and nonlinearly scattered particles should stay in the resonance approximately the same time, and there is no separation between these two types of trajectories. Figure 5(b) confirms this conclusion: We solve equation Eq. (17) with $\delta_{0}=\varepsilon^{2 / 3}$ for set of trajectories and plot these trajectories in the phase plane $(\xi, d \xi / d \tau)$. There are still some trajectories similar to trapped trajectories of the original system, but particles on these trajectories do not fulfill a single oscillation 

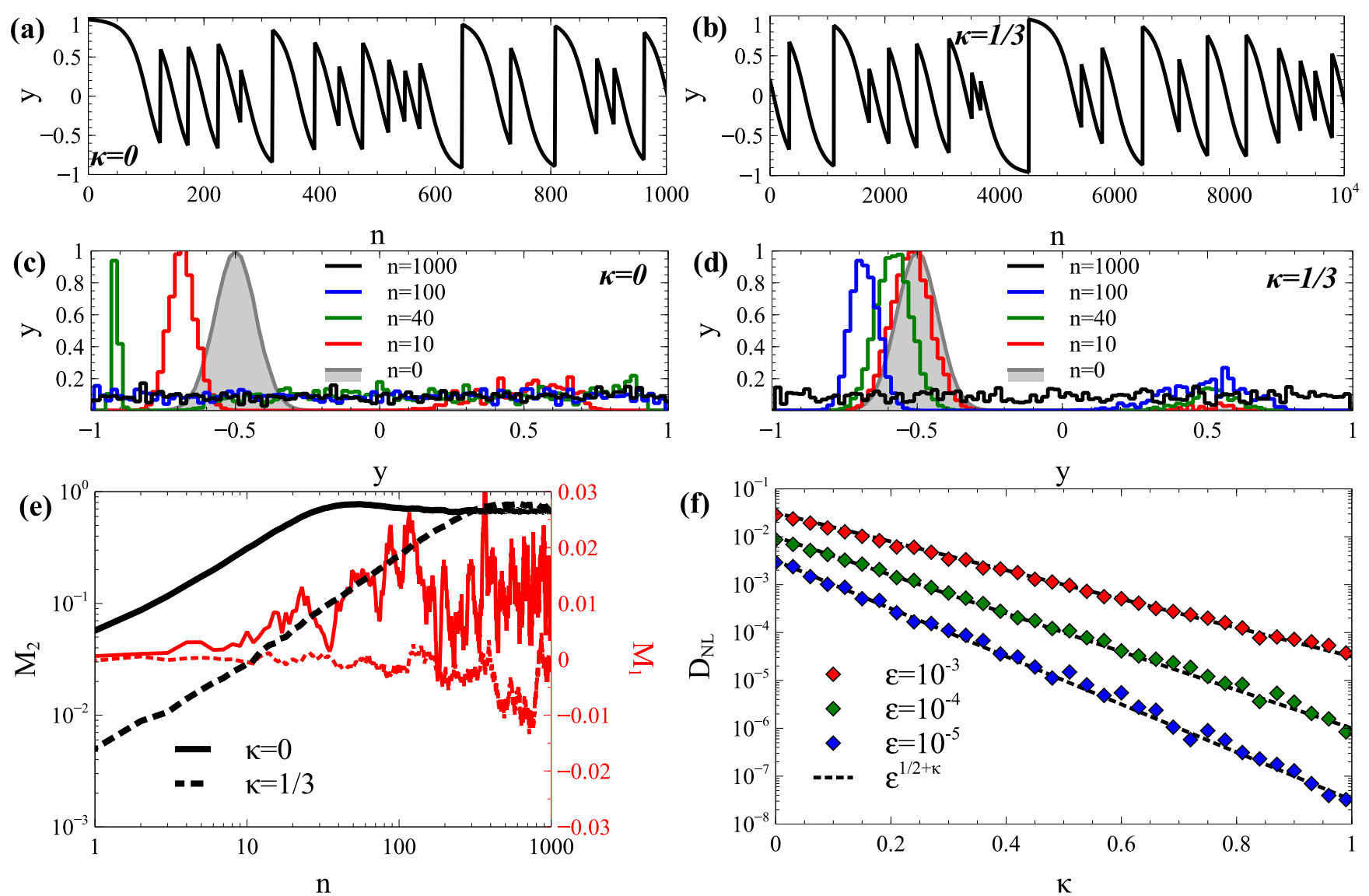

FIG. 6. Examples of $y_{n}$ trajectories described by map (18) [(a) and (b)] and evolution of $f(y)$ distribution [(c) and (d)] for two $\kappa$ values. Profiles of $\mathcal{M}_{1}(n)$ and $\mathcal{M}_{2}(n)$ for two $\kappa$ values (e). Dependence of $D_{\mathrm{NL}}$ on $\kappa$ for three $\varepsilon$ values (f).

across the resonance $d \xi / d \tau=0$, i.e., we cannot separate particles on trapped and scattered trajectories.

\section{B. Timescales of $f(\gamma)$ evolution}

To investigate how the rate of $f(\gamma)$ evolution scales with $\delta_{0}$ we use the mapping technique. Equation (15) shows that the area $S$ can be set as $\varepsilon^{1 / 2} \delta_{0}^{5 / 4}\left(1-y^{2}\right)^{5 / 4}$ where $y$ is the effective particle energy at the resonance (shifted relative to the energy where $S$ reaches maximum), i.e., different values of slow time $\sim \varepsilon t$ in Eq. (15) correspond to different $s_{R}$ values of the original system and to different resonant energies. Therefore, we can rewrite mapping (12) as

$$
y_{n+1}=y_{n}+ \begin{cases}-2 y_{n}, & \theta \in[0, \Pi] \\ -\mathcal{S}\left(y_{n}\right), & \theta \in(\Pi, 1]\end{cases}
$$

and

$$
\begin{aligned}
& \mathcal{S}=\varepsilon^{1 / 2+\kappa}\left(1-y^{2}\right)^{5 / 4} \\
& \Pi=\frac{d \mathcal{S}}{d y}=-\frac{5}{2} y \varepsilon^{1 / 2+\kappa}\left(1-y^{2}\right)^{1 / 4},
\end{aligned}
$$

where we introduce $\kappa$ as $\delta_{0}=\varepsilon^{4 \kappa / 5}$ and take into account $\mathcal{S}(-y)=\mathcal{S}(y)$ to calculate $y$ change due to trapping. Figure 6(a) shows an example of $y_{n}$ trajectory described by map (18). This trajectory repeats all features seen in the original system trajectories [see Figs. 1(d) and 4(c)]. The relaxation of the initially localized $f(y)$ distribution also repeats well the relaxation of the $f(\gamma)$ distribution: Compare Fig. 6(b) and Figs. 2(d) and 4(d).

To characterize the timescale of $f(y)$ evolution as a function of $\kappa$, we numerically integrate a large ensemble of trajectories described by map (18) for various values of $\kappa$. The initial $y_{0, i}$ values for $i=0 \cdots N$ trajectories are uniformly distributed within $[-1,1]$ range, and we calculate two characteristics: $\mathcal{M}_{1}(n)=N^{-1} \sum_{i=0 . . N}\left(y_{n, i}-y_{0, i}\right)$ and $\mathcal{M}_{2}(n)=N^{-1} \sum_{i=0 . . N}\left(y_{n, i}-y_{0, i}\right)^{2}-\mathcal{M}_{1}^{2}(n)$. Figure 6(e) shows $\mathcal{M}_{1}(n), \mathcal{M}_{2}(n)$ profiles for two $\kappa$ values: $\kappa=0$ corresponds to the system with well-distinguished populations of trapped and scattered particles (i.e., the system is far from the diffusive regime), whereas $\kappa=1 / 3$ corresponds to $\delta_{0}=\varepsilon^{4 \kappa / 5}=\varepsilon^{4 / 15}$ and this is a case with much closer timescales of the dynamics of trapped and scattered particle populations. For both values of $\kappa \mathcal{M}_{2}$ grows with $n$ and $\mathcal{M}_{1}$ oscillates around the zero.

To derive the timescale of $f(y)$ relaxation we fit the growing fragment of $\mathcal{M}_{2}(n)$ profile by the linear function $D_{\mathrm{NL}} n$ with the coefficient $D_{\mathrm{NL}}$ playing the role of a diffusion coefficient in systems with diffusive resonant interactions. Figure 6(f) shows the $D_{\mathrm{NL}}$ dependence on $\kappa$ for three $\varepsilon$ values. There is a clear scaling $D_{\mathrm{NL}} \approx \varepsilon^{1 / 2+\kappa}$, i.e., $D_{\mathrm{NL}}$ varies as $\varepsilon^{1 / 2}$ for $\kappa=0$ and as $\varepsilon^{4 / 3}$ for $\kappa=5 / 6$. This scaling may be explained by the dominating role of trappings in the $\mathcal{M}_{2}$ increase. Most of nonlinearly scattered particles change their 
energy only slightly, and the first-order contribution of this energy change goes into $\mathcal{M}_{1}$, whereas the contribution of these particles to the growth of $\mathcal{M}_{2}$ is about $\sim \mathcal{S}^{2} \sim \varepsilon^{1+2 \kappa}$. However, even a small population of trapped particles would contribute to $N^{-1} \sum_{i=0 . . N}\left(y_{n, i}-y_{0, i}\right)^{2}$ as $\sim \Pi \sim \varepsilon^{1 / 2+\kappa}$ [the number of trapped particles is about the probability of trapping, whereas these particles have $\left.y_{n, i}-y_{0, i} \sim O(1)\right]$; on the other hand, their contribution to $\mathcal{M}_{1}^{2}$ is much smaller. Thus, $\mathcal{M}_{2} \sim \Pi$ and this confirms the scaling from Fig. 6(f).

\section{Timescale of trapping acceleration}

The scaling from Fig. $6(\mathrm{~d})$ shows that $D_{\mathrm{NL}}$ not only depends on $\varepsilon$ but also scales with $\delta_{0} \sim \varepsilon^{4 \kappa / 5}$. This parameter, $\delta_{0}$, controls the effective range of energy change due to trapping and should be related to the number of complete rotations around the resonance of trapped particles, $N_{\text {trap }}$. Thus, we can derive the $\delta_{0}\left(N_{\text {trap }}\right)$ dependence and rewrite $D_{\mathrm{NL}}$ as a function of $\varepsilon$ and $N_{\text {trap }}$. We define $N_{\text {trap }}$ as the maximum number of periods of a trapped particle's rotation in the phase space [see phase portrait in Fig. 3(b)]. This parameter is quite universal: It can be determined for any particular wave-field model (see, e.g., Ref. [44] for a discussion of $N_{\text {trap }}$ values typical for whistler-mode waves observed in the Earth's radiation belts). The trapped period for Hamiltonian (14) can be written as

$$
T_{\text {trap }}(\varepsilon t)=2 \int_{\zeta_{-}}^{\zeta_{+}} \frac{d \zeta}{\dot{\zeta}}=\frac{C}{\varepsilon^{1 / 2}\left(\delta_{0}-\varepsilon^{2} t^{2}\right)^{1 / 4}},
$$

where constant $C \sim O(1)$ is determined by the distance from the separatrix in the phase portrait. The maximum time of trapped particle motion is of the order of $\sim 2 \delta_{0}^{1 / 2} / \varepsilon$. Therefore we can write for $N_{\text {trap }}$

$$
N_{\text {trap }}=\frac{2}{\varepsilon} \int_{0}^{\sqrt{\delta_{0}} / \varepsilon} \frac{d \varepsilon t}{T_{\text {trap }}(\varepsilon t)}=\frac{\delta_{0}^{3 / 4}}{\sqrt{\varepsilon}} \tilde{C} \sim \varepsilon^{3 \kappa / 5-1 / 2},
$$

where constant $\tilde{C} \sim O(1)$. Equation (20) shows that $N_{\text {trap }} \sim$ $\varepsilon^{3 \kappa / 5-1 / 2}$. For the threshold value $\kappa=5 / 6$, we get $N_{\text {trap }} \sim$ $O(1)$, i.e., indeed for $\kappa=5 / 6\left(\delta_{0} \sim \varepsilon^{2 / 3}\right)$ the number of trapping periods does not depend on $\varepsilon$ and there is no separation between trapped and scattered particles anymore.

Combining Eq. (20) and the $D_{\mathrm{NL}}$ scaling, we obtain the final scaling of the coefficient $D_{\mathrm{NL}}$,

$$
D_{\mathrm{NL}} \sim \varepsilon^{1 / 2+\kappa} \sim \varepsilon^{4 / 3} N_{\text {trap }}^{5 / 3} \sim D_{\mathrm{QL}} \varepsilon^{-2 / 3} N_{\text {trap }}^{5 / 3},
$$

where we take into account that $D_{\mathrm{QL}} \sim \varepsilon^{2}$. Therefore, $D_{\mathrm{NL}} / D_{\mathrm{QL}}=\mathcal{Q} \varepsilon^{-2 / 3} N_{\text {trap }}^{5 / 3}$ where the factor $\mathcal{Q} \sim O(1)$ is determined by the system parameters. According to this scaling, the transition between the diffusive regime $\left(N_{\text {trap }} \rightarrow 0\right)$ and the nonlinear resonant regime $\left(N_{\text {trap }} \gg 1\right)$ occurs when $N_{\text {trap }} \sim \varepsilon^{2 / 5} \ll 1$, whereas for $\kappa=5 / 6\left(N_{\text {trap }} \sim 1\right)$ we get $D_{\mathrm{NL}} / D_{\mathrm{QL}} \sim \varepsilon^{-2 / 3} \gg 1$.

However, the scaling in Eq. (21) is obtained for systems where $\varepsilon$ and $N_{\text {trap }}$ (or more precisely $\kappa$, which determines $\delta_{0}$ ) are assumed to be independent parameters that can be varied separately. But $\varepsilon$ and $N_{\text {trap }}$ are not always independent. This is especially the case in realistic systems, where whistler-mode waves often propagate in the form of short wave packets (see examples of such wave packets in observations [44] and in numerical simulations $[77,78])$. We consider this realistic situation in the next subsection.

\section{Systems with short wave packets: Shrinking of the resonant energy range}

In the Earth's magnetosphere, intense whistler-mode waves contain a fine structure of packets or subpackets, where packets and subpackets are formed by strong wave amplitude modulations (by at least a factor of $\sim 2$ ) accompanied by large and random wave phase jumps between successive packets and subpackets, allowing us to treat electron interactions with different packets and subpackets independently $[34,36,79,80]$. In particular, most long wave envelopes, characterized by an amplitude continuously remaining above $50 \mathrm{pT}$, actually consist of many shorter packets and subpackets when internal strong amplitude modulations and wave frequency and phase jumps are taken into account $[36,53,80]$. The overwhelming majority of such intense parallel propagating whistler-mode wave packets and subpackets observed in the magnetosphere have a short length of $\beta<10$ wave periods, with much more rare long packets and subpackets reaching $\beta \sim 100-300$ periods [36,44,81]. Short packets of length $\beta<10$ are likely often produced by wave superposition $[53,77,81]$ and their length $\beta$ is mostly independent of their amplitude $\varepsilon$. Packets of intermediate length $\beta \sim 10-30$ are probably partly formed by trapping-induced amplitude modulation that depends on $\varepsilon^{-1 / 2}$, but statistical observations show that all packets are distributed over a wide range of $\beta$ values for any given $\varepsilon$ $[34,53,77]$, allowing to treat $\beta$ and $\varepsilon$ as independent parameters to leading order.

Taking into account that first-order cyclotron resonance between whistler-mode waves and $<1 \mathrm{MeV}$ electrons occurs for waves and electrons moving in opposite directions, the time interval of trapped particle motion is limited to $\sim 2 \pi \beta / \Omega_{0}$ (this is a simplified estimate of $\sim 2 \pi \beta / k\left|\partial \omega / \partial k-v_{R}\right|$ with $v_{R}=\left(\omega-\Omega_{0} / \gamma\right) / k$ and $\partial \omega / \partial k \sim \omega / k$ for not-too-high electron energy $\gamma \sim 1$; see details in Ref. [44]), whereas one period of trapped electron oscillation is $T_{\text {trap }} \sim 2 \pi \varepsilon^{-1 / 2} / \Omega_{0}$ for typical resonant energies [6]. Therefore, $N_{\text {trap }}$ can be expressed as a function of the independent parameters $\beta$ and $\varepsilon$ of short wave packets, giving $N_{\text {trap }} \sim \beta \varepsilon^{1 / 2}$ (see also Ref. [44]).

In the case of short wave packets, although the magnitude of $S$ remains $\sim \varepsilon^{1 / 2}$, the limited packet size can lead to important changes as compared with the scaling obtained in Eq. (21) of the preceding subsection in the ideal case of infinitely long wave packets or waves. Indeed, a given short wave packet occupies only a limited latitudinal extent along a magnetic field line. Equation (5) and the conservation of $h$, which relate latitude of resonance to electron energy imply that this limited latitudinal extent directly corresponds to a limited energy range for electron resonance with this packet during one bounce period. This limited energy range of actually resonant electrons represents a fraction $\ell<1$ of the total energy range of particles potentially reaching resonance with a wave packet over the full length of the magnetic field line. Electron trapping by a short packet leads to a smaller energy change than trapping by an ideal infinitely long wave packet, because the electron is released from trapping faster, corresponding to a reduction of its energy change by a factor 
$\sim \ell$. Besides, if the infinitely long wave packet resonates with all electrons arriving to the latitude of resonance, then a short wave packet occupies only a fraction $\ell$ of the magnetic field line and many electrons arriving to their latitude of resonance would not meet this wave packet. Therefore, if short wave packets are rare (not appearing in close succession) and occur only approximately once every bounce period, then this corresponds to an additional reduction of the occurrence rate of resonant interaction by a factor $\sim \ell<1$ as compared with both the case of a close succession of short packets and the ideal case of infinitely long packets assumed in the preceding subsection.

Accordingly, let us consider a realistic situation where the magnitude of $\mathcal{S} \sim \sqrt{\varepsilon}$ remains the same, but the range of nonlinear resonant interactions [formally the $\varepsilon t$ range in Eq. (14)] shrinks. There are two kinds of systems with an entire resonant range $y \in[-1,1]$, but where $\mathcal{S}$ is not equal to zero only for $y \in[-\ell, \ell]$, with $\ell=\sqrt{\delta_{0}}$. In systems of the first kind, we center $\mathcal{S}$ around a $\bar{y}_{n}$ randomly generated at each map iteration around $y_{n}$, i.e., for each iteration there is a finite change of $y_{n}$. This type of mapping mimics electron resonant interactions with a set of short wave packets that would fill the entire magnetic field lines but which cannot trap electrons for a long time because of the limited packet duration. In the system of the second kind, we center $\mathcal{S}$ around a randomly generated $\bar{y}_{n}$, but we do not control the position of $\bar{y}_{n}$ relative to $y_{n}$ and some map iterations can occur without any change of $y_{n}$ because $y_{n}$ is outside the $\mathcal{S} \neq 0$ range. This type of mapping mimics electron resonant interaction with rare short wave-packets propagating with some time separation. It corresponds to a situation where, during each bounce period, only one wave packet is present and many electrons reach the latitude of cyclotron resonance without encountering this intense packet there. For both systems, the mapping (18) can be rewritten as

$$
y_{n+1}=y_{n}+ \begin{cases}\bar{y}_{n}-2 y_{n}, & \theta \in[0, \Pi] \\ -\mathcal{S}_{n}\left(y_{n}\right), & \theta \in(\Pi, 1]\end{cases}
$$

and

$$
\begin{aligned}
\mathcal{S}_{n} & =\varepsilon^{1 / 2}\left[\ell^{2}-\left(y-\bar{y}_{n}\right)^{2}\right]^{5 / 4} \\
\Pi & =\frac{d \mathcal{S}_{n}}{d y}=-\frac{5}{2}\left(y-\bar{y}_{n}\right) \varepsilon^{1 / 2}\left[\ell^{2}-\left(y-\bar{y}_{n}\right)^{2}\right]^{1 / 4},
\end{aligned}
$$

where $\bar{y}_{n}=y_{n}+\ell R$ for the system of the first kind and $\bar{y}_{n}=$ $-1+\ell+2(1-\ell) R$ for the system of the second kind; here $R$ is a random number with a uniform distribution within $[-1,1]$ (note for $\bar{y}_{n}=y_{n}+\ell R$ we also control that $\mathcal{S} \neq 0$ range does not cross $y \pm 1$ boundaries). An important property of both systems is that for small $\ell$ trappings are possible for any $y_{n}$ values, whereas for $\ell=1$ [the initial map given by Eq. (18)] trapping is possible only for $y_{n}<0$. Figures 7(a) and 7(b) shows a set of sample trajectories for each of the two kinds of systems. The rate of change of $y$ is going down as $\ell$ decreases, and this effect is stronger for the system of the second kind [Fig. 7(b)].

For these two kinds of systems we set the range of $\ell$ and for each $\ell$ value calculate $M_{2}(n)$. Figures 7(c) and 7(d) shows examples of $M_{2}$ profiles. We fit the growing fragment of $\mathcal{M}_{2}(n)$ by $D_{\mathrm{NL}} n$, and Figs. 7(e) and 7(f) shows the $D_{\mathrm{NL}}$ dependence on $\ell$. For both systems, $D_{\mathrm{NL}}$ scales with $\ell$ as $D_{\mathrm{NL}} \sim \varepsilon^{1 / 2} \ell^{\eta}$. But $\eta \approx 7 / 2$ for the system of the first kind where resonances occur at each iteration, whereas $\eta \approx 9 / 2$ for the system of the second kind, where resonance occurrence is decreased by a factor $\ell \ll 1$. Taking into account that $\ell=\sqrt{\delta_{0}}$, we can rewrite Eq. (20) as $N_{\text {trap }} \sim \ell^{3 / 2} / \sqrt{\varepsilon}$. Therefore, for $D_{\mathrm{NL}}$ scaling with $N_{\text {trap }}$ we have $D_{\mathrm{NL}} \sim \varepsilon^{1 / 2} \ell^{\eta} \sim \varepsilon^{1 / 2+\eta / 3} N_{\text {trap }}^{2 \eta / 3}$ and

$$
D_{\mathrm{NL}} \sim \begin{cases}\varepsilon^{5 / 3} N_{\text {trap }}^{7 / 3}, & \eta=7 / 2 \\ \varepsilon^{2} N_{\text {trap }}^{3}, & \eta=9 / 2\end{cases}
$$

for the two kinds of systems.

A general scaling, including both the results in Eq. (23) and in Eq. (21), is

$$
D_{\mathrm{NL}} \sim \varepsilon^{1 / 2+\eta / 3} N_{\text {trap }}^{2 \eta / 3} \sim \varepsilon^{1 / 2+2 \eta / 3} \beta^{2 \eta / 3} .
$$

For $\eta=5 / 2$, Eq. (24) allows us to recover the initial scaling from Eq. (21), which corresponds to the ideal case of electron interactions with infinitely long wave packets and waves present all the time along magnetic field lines. In the more realistic situation of short wave packets, two different kinds of systems are possible, corresponding to $\eta=$ $7 / 2$ and $\eta=9 / 2$. For the system of the first kind, we get $D_{\mathrm{NL}} / D_{\mathrm{QL}} \sim \varepsilon^{-1 / 3} N_{\text {trap }}^{7 / 3} \sim \varepsilon^{5 / 6} \beta^{7 / 3}$. In this case, $D_{\mathrm{NL}} / D_{\mathrm{QL}} \sim$ $O$ (1) requires that $N_{\text {trap }} \sim \varepsilon^{1 / 7}$ and $\beta \sim \varepsilon^{-5 / 14}$. For realistic whistler-mode wave amplitudes $\varepsilon \in\left[3 \times 10^{-4}, 3 \times 10^{-3}\right]$ in the Earth's radiation belts [53], the transition between the regimes of quasilinear diffusion and nonlinear resonant interaction should therefore occur approximately for $N_{\text {trap }}>0.35$ and $\beta>10$. In the system of the second kind, we have $D_{\mathrm{NL}} / D_{\mathrm{QL}} \sim N_{\text {trap }}^{3} \sim \beta^{3} \varepsilon^{3 / 2}$. In this case, $D_{\mathrm{NL}} / D_{\mathrm{QL}} \sim O(1)$ corresponds to $N_{\text {trap }} \sim 1$ and $\beta \sim \varepsilon^{-1 / 2}$. Thus, for this system of very rare short packets, the transition between the regimes of quasilinear diffusion and nonlinear resonant interaction should occur around $N_{\text {trap }} \sim 1$ and approximately for $\beta>20$ - i.e., near the $\kappa=5 / 6$ threshold value when $\delta_{0} \sim \varepsilon^{2 / 3}$ and $\ell \sim \varepsilon^{1 / 3}$.

Note that the $D_{\mathrm{NL}}$ scaling in Eq. (24) for the first kind of system with short wave packets, corresponding to $\eta=7 / 2$, is valid only when cyclotron resonance with wave packets is always available, i.e., when electrons with any energy within the resonant range interact with the wave once (or more frequently) per bounce period. In practice, however, intense wave packets are not always present $[36,44]$. Nevertheless, the overwhelming majority of wave packets interact with electrons independently of the other packets [80], and their very large population is mostly randomly distributed in time. Consequently, over a sufficiently long time period, all latitudes of resonance with all electron energies should be uniformly reached by packets in each $(\beta, \varepsilon)$ range. In this limit, the time-averaged $\left\langle D_{\mathrm{NL}}\right\rangle$ can be simply obtained by a double integration over the full measured distribution of $(\beta>0, \varepsilon>0)$ packets of $D_{\mathrm{NL}}$, weighted by the measured temporal occurrence rate (taking into account packet duration $\beta$ ) of $(\beta, \varepsilon)$ packets $[36,44,81]$. Finally, $\left\langle D_{\mathrm{NL}}\right\rangle /\left\langle D_{\mathrm{QL}}\right\rangle$ can be obtained by dividing $\left\langle D_{\mathrm{NL}}\right\rangle$ by the integral over $\varepsilon$ of $D_{\mathrm{QL}} \sim \varepsilon^{2}$ weighted by the measured temporal occurrence rate of $\varepsilon$ packets. In addition, $D_{\mathrm{NL}} / D_{\mathrm{QL}}$ should be considered as $\neq 1$ in the above integration only for $\varepsilon$ values above the threshold for nonlinear 

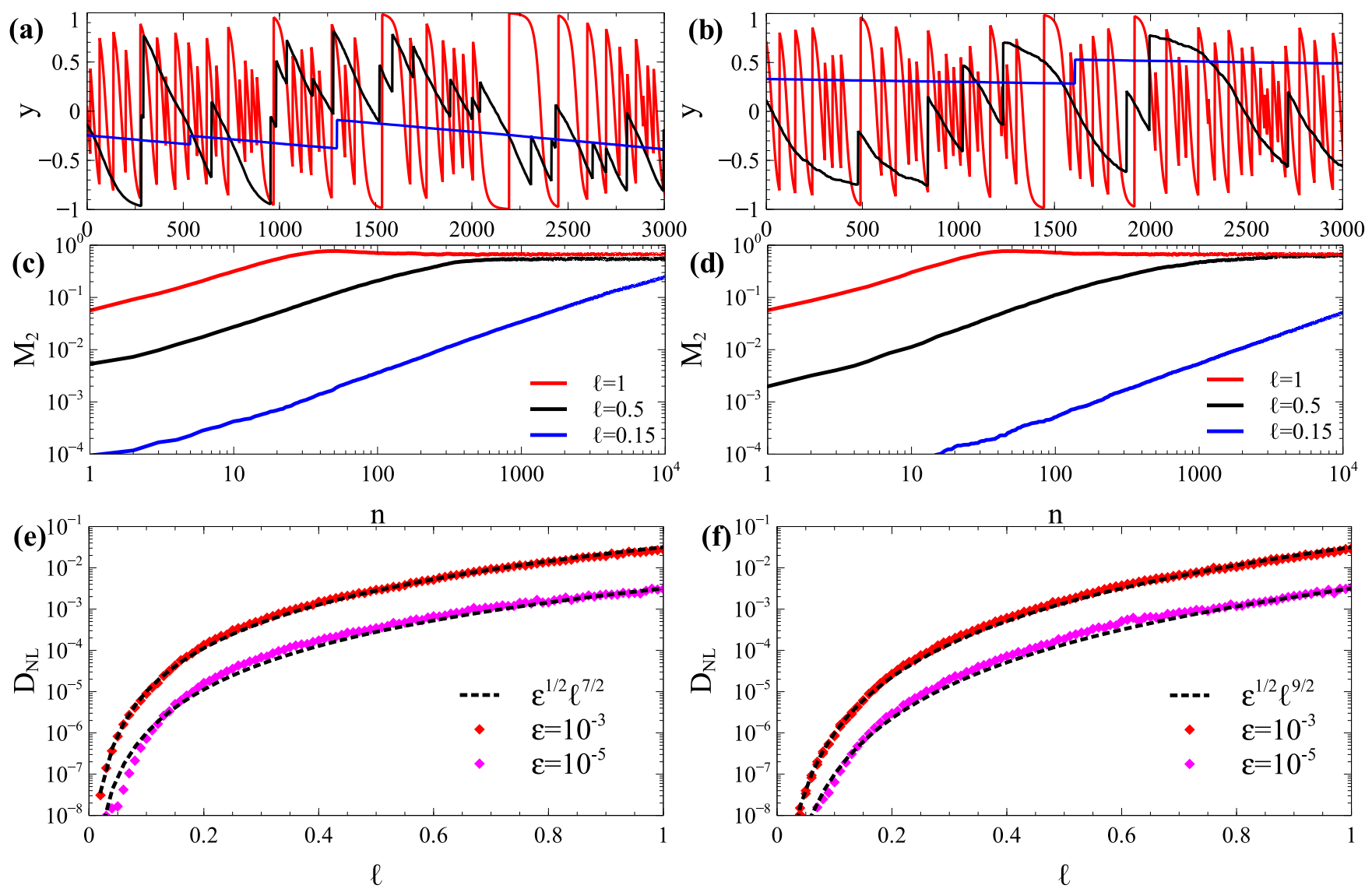

FIG. 7. Three trajectories for different $\ell$ values [see color codes in panels (c) and (d)] for systems of two different kinds [(a) and (b)]. Examples of $M_{2}$ profiles for these two systems [(c) and (d)]. Dependencies of $D_{\mathrm{NL}}$ on $\ell$ for the two systems and different $\varepsilon$ [(d) and (f)]. Left column [(a), (c), and (e)] shows results for the system of the first kind: the entire resonant range $y \in[-1,1]$, but $S$ is not equal to zero only for $[-\ell, \ell]$ appearing always around the particle location, i.e., there is a resonant interaction at each iteration. The right column [(b), (d), and (f)] shows results for the system of the second kind: the entire resonant range $y \in[-1,1]$, but $S$ is not equal to zero only over $[-\ell, \ell]$ appearing at random locations, not necessarily near the particle location, i.e., there are iterations without change of particle $y$.

effects $[81,82]$. For lower amplitudes, one can simply assume $D_{\mathrm{NL}} / D_{\mathrm{QL}} \approx 1$.

\section{DISCUSSION AND CONCLUSION}

In this study, we investigate the transition between two regimes of resonant electron interactions with whistler-mode waves: quasilinear diffusion and nonlinear resonant interaction. The typical effects of these two regimes are very different: Diffusive scattering results in small energy changes with zero mean value, whereas nonlinear resonant interactions result in energy drifts (due to phase bunching) or large jumps (due to phase trapping). This difference makes it impossible to directly compare energy changes for these two regimes, i.e., energy drifts and jumps cannot be compared with the diffusion rates. Therefore, a more general (nonlocal, i.e., independent on the initial energy value) characteristic should be determined to compare these two regimes. For such characteristic, we choose the typical timescale of relaxation of the energy distribution function $f(\gamma)$, which is determined along a single resonant curve $h=$ const. This characteristic describes the entire energy, pitch-angle range for a given $h=\gamma-[\omega / 2 \Omega(0)]\left(\gamma^{2}-1\right) \sin ^{2} \alpha_{0}$ and wave frequency, i.e., it is an integral characteristic that simplifies the system description. The relaxation of the $f(\gamma)$ distribution depends on the initial $f(\gamma)$ and contains many elements that are different for these two regimes (e.g., formation of local maxima due to the phase trapping acceleration). Therefore, to further simplify the description of this evolution and reduce it to a single parameter, we restrict our consideration to the dynamics of $f(\gamma)$ dispersion $\mathcal{M}_{2}$ that increases with the number of resonance crossings and finally saturates. This parameter $\mathcal{M}_{2}(n)$ is the unique characteristic for the diffusive resonant regime, but for nonlinear resonant interactions there are also $\mathcal{M}_{1}(n)$ and higher-order moments. Thus, some justification for taking only $\mathcal{M}_{2}$ into account should be given. The first moment $\mathcal{M}_{1}(n)=V n$ scales with $\varepsilon$ as $V \sim \mathcal{S} \sim$ $\varepsilon^{1 / 2+\kappa}$, and the same scaling can be obtained for $\mathcal{M}_{2}(n)=$ $D_{\mathrm{NL}} n$ with $D_{\mathrm{NL}} \sim \mathcal{S} \sim \varepsilon^{1 / 2+\kappa}$. The beginning of the evolution (relaxation) of the distribution $f(\gamma)$ is dominated by trapping effects contributing to $\mathcal{M}_{2}$, because $\delta \gamma^{2} \sim D_{\mathrm{NL}} n$ is larger than $\delta \gamma^{2} \sim V^{2} n^{2}$ for small $n$. The threshold value of $n$ such that drifts become more important than trappings is $n^{*} \sim D_{\mathrm{NL}} / V^{2} \sim \varepsilon^{-1 / 2-\kappa}$. During this number of resonant interactions the first trapped particles would be drifted back to their initial energies, because $\Delta \gamma_{\text {trap }} / \Delta \gamma_{\text {scat }} \sim 1 / S \sim \varepsilon^{-1 / 2-\kappa}$ and $n^{*}\left(\Delta \gamma_{\text {trap }} / \Delta \gamma_{\text {scat }}\right) \sim O(1)$. The drift effects may control the relaxation of $f(\gamma)$ only after the main relaxation 
stage is finished [when the initially trapped particles reach back their initial energies, the first large cycle of trappingscattering would be finished; see, e.g., example of such cycles in Figs. 1(d)]. Thus, we indeed can use $\mathcal{M}_{2}(n)$ to characterize the main stage of $f(\gamma)$ relaxation. Note that for the regime of nonlinear resonant interaction $\mathcal{M}_{2}(n)$ does not really describe a diffusion (because such nonlinear interactions may result in formation of transient local gradients of $f(\gamma)$ absent in a diffusive evolution), but it shows how quickly the dispersion of $f(\gamma)$ grows due to trapping and nonlinear scattering effects. However, the rate $D_{\mathrm{NL}}=d M_{2}(n) / d n$ can still be considered as an approximation of the diffusion rate corresponding to a diffusive $f(\gamma)$ relaxation with the same final result as nonlinear interactions and with the same timescale.

In the Earth's magnetosphere, whistler-mode waves mainly propagate in the form of wave packets, and most of the observed parallel propagating intense wave packets are short, containing $\beta<10-30$ wave periods $[36,44]$. The relations obtained here, connecting $D_{\mathrm{NL}} / D_{\mathrm{QL}}$ with wave-packet amplitude $\varepsilon$ and number of trapped electron oscillations $N_{\text {trap }}$ or packet length $\beta$, can be considered as inverse scaling laws for the timescale of $f(\gamma)$ relaxation-although such a consideration simplifies the details of $f(\gamma)$ evolution. The main potential application of these scaling laws is to allow including the leading-order effect of nonlinear resonant interactions into global models of radiation belt dynamics relying on the Fokker-Planck diffusion equation. This equation describes the evolution of $f(\gamma)$ driven by diffusion on a typical timescale $\sim 1 / D_{\mathrm{QL}}$. This timescale is close to the observed timescales of electron flux dynamics in the Earth's radiation belts during quiet time periods characterized by not-to-high wave intensity (e.g., Refs. [83-85]). However, during more disturbed periods with a high occurrence rate of intense wave packets [10], the observed electron flux evolution could be faster than the evolution predicted by a diffusion model (see discussions in, e.g., Refs. $[14,36,86]$ ). Our scaling laws of $D_{\mathrm{NL}} / D_{\mathrm{QL}}$ allow for a simple renormalization of the quasilinear diffusion rates based on the observed occurrence rate of intense waves resonantly interacting with electrons in the nonlinear regime. An important ingredient of such a renormalization is to take into account not only the wave intensity $\left(\sim \varepsilon^{2}\right)$ but also the actual fine structure of intense wave packets $[34,53,79,80]$. We can take into account these fine structure effects via the parameter $N_{\text {trap }}$, the number of periods of trapped particle motion. Although $N_{\text {trap }}$ is not a directly measured wave characteristic, this parameter can include effects of wave-packet shortness $[36,44,54,80]$ and effects of wave coherence destruction [34,80,87]. Alternatively, we can take into account the fine structure of whistler-mode waves via the parameter $\beta$, the wave-packet length in number of wave periods, which can be directly measured together with $\varepsilon$ [44]. For resonant interactions with $<1-\mathrm{MeV}$ electrons, we get $D_{\mathrm{NL}} / D_{\mathrm{QL}} \sim$ $\varepsilon^{-3 / 2+2 \eta / 3} \beta^{2 \eta / 3}$ with a value of $\eta \in[5 / 2,9 / 2]$ that depends on assumptions concerning the wave-packet distribution in the system. For the rare very long wave packets with $\beta \sim 10^{2}$ and high wave amplitude $\varepsilon \sim 10^{-3}$ we obtain $D_{\mathrm{NL}} / D_{\mathrm{QL}} \sim$ 650 for $\eta=5 / 2$ and $D_{\mathrm{NL}} / D_{\mathrm{QL}} \sim 150$ for $\eta=7 / 2$, but the time-weighted occurrence rate of such long and intense wave packets varies between $\sim 0.5 \times 10^{-3}$ and $\sim 2 \times 10^{-3}$ from mildly to strongly active geomagnetic conditions [36,44]. The most realistic case is a long succession of short wave packets (corresponding to $\eta=7 / 2$ ) and for wave amplitudes above an approximate threshold $\varepsilon \geqslant 10^{-3}$ for nonlinear interaction [81,82]. In this case, integrating over $\beta \sim 2-200$ and $\varepsilon \sim 10^{-3}-10^{-2}$ expression (24) for $D_{\mathrm{NL}}$, weighted by the measured occurrence rate of a large statistics of such packets [81] (with average packet power roughly equal to half its peak power, as in Ref. [78]), gives $\left\langle D_{\mathrm{NL}}\right\rangle_{\beta, \varepsilon} /\left\langle D_{\mathrm{QL}}\right\rangle_{\beta, \varepsilon} \sim$ 3 . For smaller amplitudes $\varepsilon<10^{-3}$, representing $\approx 15 \%$ of the wave power [81], we should have $D_{\mathrm{NL}} \sim D_{\mathrm{QL}}$, giving a final approximate time-averaged ratio $\left\langle D_{\mathrm{NL}}\right\rangle_{\beta, \varepsilon} /\left\langle D_{\mathrm{QL}}\right\rangle_{\beta, \varepsilon} \sim$ 2.5. These estimates suggest that over relatively long time periods ( $\sim$ days $)$, nonlinear interactions should only slightly speed up the evolution of electron fluxes in comparison with the diffusive evolution for the same time-averaged wave intensity. However, a more accurate parametrical study would be needed to determine for what energies, pitch angles, and geomagnetic conditions the contribution of nonlinear interactions maximizes. It is also worth noting that the present comparison of relatively long timescales of electron distribution relaxation does not contradict the fact that only the regime of nonlinear resonant interaction can explain various short-lived events of rapid electron acceleration or loss, which occur much faster than the diffusive evolution (e.g., Refs. [12,14]).

To conclude, we have investigated the transition between two regimes of resonant interaction of electrons and whistler-mode waves in the Earth's radiation belts: quasilinear diffusion and nonlinear resonant interaction (including effects of phase trapping and phase bunching). To characterize both regimes within the same framework, we have introduced the typical timescale $1 / D$ of evolution of the electron energy distribution on the resonance curve. This timescale characterizes the increase of the distribution dispersion for both diffusive $\left(1 / D_{\mathrm{QL}}\right)$ and nonlinear $\left(1 / D_{\mathrm{NL}}\right)$ relaxations of the electron distribution. For the diffusive relaxation $D_{\mathrm{QL}} \sim \varepsilon^{2}$ where $\varepsilon=B_{w} / B_{0}$ is the normalized wave amplitude. Thus, we investigated how $D_{\mathrm{NL}}$ depends on $\varepsilon$ and on the characteristics of intense wave packets, which are described by $N_{\text {trap }}$, the number of periods in trapped motion, or $\beta$, the wave-packet size $\left(N_{\text {trap }} \sim \beta \varepsilon^{1 / 2}\right.$ when $\left.\beta<1 / \varepsilon\right)$ and/or to the closeness of the wave-packet amplitude to its threshold value required for nonlinear resonances $\left(N_{\text {trap }} \sim \delta_{0}^{3 / 4} \varepsilon^{-1 / 2}\right.$ with $\delta_{0} \rightarrow 1$ for large amplitude packets). The obtained scaling is $D_{\mathrm{NL}} / D_{\mathrm{QL}} \sim$ $\varepsilon^{-3 / 2}(\varepsilon \beta)^{2 \eta / 3}$ with $\eta$ varying from $5 / 2$ (when we compare electron distribution relaxation due to diffusion and nonlinear interactions over the same energy range) to $9 / 2$ (when diffusion acts over a wide energy range, whereas nonlinear interactions are very rare and occur in a narrower energy range). These scalings can be used for generalizing the existing radiation belt models and for an evaluation of the importance of nonlinear interactions for realistic distributions of wave characteristics.

\section{ACKNOWLEDGMENTS}

The work of A.V.A., A.I.N., and A.A.V. was supported by the Russian Science Foundation, Project No. 19-12-00313. 
[1] A. A. Vedenov, E. Velikhov, and R. Sagdeev, Nucl. Fusion Suppl. 2, 465 (1962).

[2] W. E. Drummond and D. Pines, Nucl. Fusion Suppl. 3, 1049 (1962).

[3] T. O’Neil, Phys. Fluids 8, 2255 (1965).

[4] R. K. Mazitov, J. Appl. Mech. Tech. Phys. 6, 22 (1965).

[5] C. F. Kennel and F. Engelmann, Phys. Fluids 9, 2377 (1966).

[6] V. I. Karpman, Space Sci. Rev. 16, 361 (1974).

[7] C. F. Kennel and H. E. Petschek, J. Geophys. Res. 71, 1 (1966).

[8] D. Nunn, J. Plasma Phys. 6, 291 (1971).

[9] V. I. Karpman, J. N. Istomin, and D. R. Shklyar, Plasma Phys. 16, 685 (1974).

[10] R. M. Thorne, W. Li, B. Ni, Q. Ma, J. Bortnik, L. Chen, D. N. Baker, H. E. Spence, G. D. Reeves, M. G. Henderson, C. A. Kletzing, W. S. Kurth, G. B. Hospodarsky, J. B. Blake, J. F. Fennell, S. G. Claudepierre, and S. G. Kanekal, Nature (Lond.) 504, 411 (2013).

[11] W. Li, R. M. Thorne, Q. Ma, B. Ni, J. Bortnik, D. N. Baker, H. E. Spence, G. D. Reeves, S. G. Kanekal, J. C. Green, C. A. Kletzing, W. S. Kurth, G. B. Hospodarsky, J. B. Blake, J. F. Fennell, and S. G. Claudepierre, J. Geophys. Res. 119, 4681 (2014).

[12] O. V. Agapitov, A. V. Artemyev, D. Mourenas, F. S. Mozer, and V. Krasnoselskikh, Geophys. Res. Lett. 42, 10 (2015).

[13] J. C. Foster, P. J. Erickson, D. N. Baker, S. G. Claudepierre, C. A. Kletzing, W. Kurth, G. D. Reeves, S. A. Thaller, H. E. Spence, Y. Y. Shprits, and J. R. Wygant, Geophys. Res. Lett. 41, 20 (2014).

[14] L. Gan, W. Li, Q. Ma, A. V. Artemyev, and J. M. Albert, Geophys. Res. Lett. 47, e90749 (2020).

[15] D. Mourenas, A. V. Artemyev, O. V. Agapitov, F. S. Mozer, and V. V. Krasnoselskikh, J. Geophys. Res. 121, 4498 (2016).

[16] A. Voshchepynets, A. Volokitin, V. Krasnoselskikh, and C. Krafft, J. Geophys. Res.: Space Phys. 122, 3915 (2017).

[17] I. V. Kuzichev, I. Y. Vasko, A. Rualdo Soto-Chavez, Y. Tong, A. V. Artemyev, S. D. Bale, and A. Spitkovsky, Astrophys. J. 882, 81 (2019).

[18] S. M. Shaaban, M. Lazar, P. H. Yoon, S. Poedts, and R. A. López, Mon. Not. R. Astron. Soc. 486, 4498 (2019).

[19] P. Veltri and G. Zimbardo, J. Geophys. Res. 98, 13335 (1993).

[20] A. M. Bykov and R. A. Treumann, Astron. Astrophys. Rev. 19, 42 (2011).

[21] C. A. Kletzing, J. Geophys. Res. 99, 11095 (1994).

[22] Y. Shen and D. J. Knudsen, J. Geophys. Res.: Space Phys. 125, e27291 (2020).

[23] A. A. Andronov and V. Y. Trakhtengerts, Geomag. Aeron. 4, 233 (1964).

[24] L. R. Lyons and D. J. Williams, Quantitative Aspects of Magnetospheric Physics (D. Reidel, Dordrecht, 1984.

[25] V. Y. Trakhtengerts and M. J. Rycroft, Whistler and Alfvén Mode Cyclotron Masers in Space (Cambridge University Press, Cambridge, 2008)

[26] D. Le Queau and A. Roux, Sol. Phys. 111, 59 (1987).

[27] V. I. Karpman and D. R. Shkliar, Plan. Sp. Sci. 25, 395 (1977).

[28] D. R. Shklyar, Sov. Phys. JETP 53, 1197 (1981).

[29] J. M. Albert, Phys. Fluids B 5, 2744 (1993).

[30] A. G. Demekhov, V. Y. Trakhtengerts, M. J. Rycroft, and D. Nunn, Geomag. Aeron. 46, 711 (2006).

[31] A. G. Demekhov, V. Y. Trakhtengerts, M. Rycroft, and D. Nunn, Geomag. Aeron. 49, 24 (2009).
[32] Y. Omura, N. Furuya, and D. Summers, J. Geophys. Res. 112, A06236 (2007).

[33] J. M. Albert, J. Geophys. Res. 115, A00F05 (2010).

[34] X. Tao, J. Bortnik, J. M. Albert, R. M. Thorne, and W. Li, J. Atmos. Sol. Terr. Phys. 99, 67 (2013).

[35] O. Allanson, C. E. J. Watt, H. Ratcliffe, H. J. Allison, N. P. Meredith, S. N. Bentley, J. P. J. Ross, and S. A. Glauert, J. Geophys. Res.: Space Phys. 125, e27949 (2020).

[36] D. Mourenas, X.-J. Zhang, A. V. Artemyev, V. Angelopoulos, R. M. Thorne, J. Bortnik, A. I. Neishtadt, and A. A. Vasiliev, J. Geophys. Res. 123, 4979 (2018).

[37] C. M. Cully, J. W. Bonnell, and R. E. Ergun, Geophys. Res. Lett. 35, L17S16 (2008).

[38] A. V. Artemyev, O. Agapitov, D. Mourenas, V. Krasnoselskikh, V. Shastun, and F. Mozer, Space Sci. Rev. 200, 261 (2016).

[39] E. Tyler, A. Breneman, C. Cattell, J. Wygant, S. Thaller, and D. Malaspina, Geophys. Res. Lett. 46, 2328 (2019).

[40] S. Benkadda, A. Sen, and D. R. Shklyar, Chaos 6, 451 (1996).

[41] A. V. Artemyev, A. I. Neishtadt, and A. A. Vasiliev, Phys. Plasmas 27, 042902 (2020).

[42] D. Vainchtein, X. J. Zhang, A. V. Artemyev, D. Mourenas, V. Angelopoulos, and R. M. Thorne, J. Geophys. Res.: Space Phys. 123, 8149 (2018).

[43] W. Li, J. Bortnik, R. M. Thorne, and V. Angelopoulos, J. Geophys. Res. 116, A12205 (2011).

[44] X. J. Zhang, R. Thorne, A. Artemyev, D. Mourenas, V. Angelopoulos, J. Bortnik, C. A. Kletzing, W. S. Kurth, and G. B. Hospodarsky, J. Geophys. Res.: Space Phys. 123, 5379 (2018).

[45] B. W. Sheeley, M. B. Moldwin, H. K. Rassoul, and R. R. Anderson, J. Geophys. Res. 106, 25631 (2001).

[46] J. M. Albert, X. Tao, and J. Bortnik, in Dynamics of the Earth's Radiation Belts and Inner Magnetosphere, edited by D. Summers, I. U. Mann, D. N. Baker, and M. Schulz (American Geophysical Union, Washington, DC, 2013).

[47] D. R. Shklyar and H. Matsumoto, Surv. Geophys. 30, 55 (2009).

[48] A. V. Artemyev, A. I. Neishtadt, D. L. Vainchtein, A. A. Vasiliev, I. Y. Vasko, and L. M. Zelenyi, Commun. Nonlin. Sci. Numer. Simul. 65, 111 (2018).

[49] A. Neishtadt, J. Appl. Math. Mech. 39, 594 (1975).

[50] A. P. Itin, A. I. Neishtadt, and A. A. Vasiliev, Physica D 141, 281 (2000).

[51] W. Li, R. M. Thorne, J. Bortnik, Y. Y. Shprits, Y. Nishimura, V. Angelopoulos, C. Chaston, O. Le Contel, and J. W. Bonnell, Geophys. Res. Lett. 38, L14103 (2011).

[52] S. Teng, X. Tao, and W. Li, Geophys. Res. Lett. 46, 3607 (2019).

[53] X. J. Zhang, D. Mourenas, A. V. Artemyev, V. Angelopoulos, W. S. Kurth, C. A. Kletzing, and G. B. Hospodarsky, Geophys. Res. Lett. 47, e88853 (2020).

[54] R. Hiraga and Y. Omura, Earth Planets Space 72, 21 (2020).

[55] A. V. Artemyev, A. I. Neishtadt, and A. A. Vasiliev, Physica D 393, 1 (2019).

[56] P. J. Palmadesso, Phys. Fluids 15, 2006 (1972).

[57] H. Karimabadi, K. Akimoto, N. Omidi, and C. R. Menyuk, Phys. Fluids B 2, 606 (1990).

[58] B. Cai, Y. Wu, and X. Tao, Geophys. Res. Lett. 47, e87991 (2020).

[59] A. V. Artemyev, A. I. Neishtadt, J. M. Albert, L. Gan, W. Li, and Q. Ma, Phys. Plasmas 28, 052902 (2021). 
[60] J. M. Albert, A. V. Artemyev, W. Li, L. Gan, and Q. Ma, J. Geophys. Res.: Space Phys. 126, e2021JA029216 (2021).

[61] A. V. Artemyev, A. A. Vasiliev, D. Mourenas, A. I. Neishtadt, O. V. Agapitov, and V. Krasnoselskikh, Phys. Plasmas 22, 112903 (2015).

[62] V. I. Arnold, V. V. Kozlov, and A. I. Neishtadt, Mathematical Aspects of Classical and Celestial Mechanics, 3rd ed., Dynamical Systems III. Encyclopedia of Mathematical Sciences (Springer-Verlag, New York, 2006)

[63] O. V. Agapitov, A. Artemyev, V. Krasnoselskikh, Y. V. Khotyaintsev, D. Mourenas, H. Breuillard, M. Balikhin, and G. Rolland, J. Geophys. Res. 118, 3407 (2013).

[64] O. V. Agapitov, D. Mourenas, A. V. Artemyev, F. S. Mozer, G. Hospodarsky, J. Bonnell, and V. Krasnoselskikh, J. Geophys. Res.: Space Phys. 123, 297 (2018).

[65] M. Hikishima, S. Yagitani, Y. Omura, and I. Nagano, J. Geophys. Res.: Space Phys. 114, A01203 (2009).

[66] Y. Katoh and Y. Omura, Earth Planets Space 68, 192 (2016).

[67] A. G. Demekhov, U. Taubenschuss, M. Hanzelka, and O. Santolík, Geophys. Res. Lett. 47, e86958 (2020).

[68] Y. Omura, Earth Planets Space 73, 95 (2021).

[69] X. Tao, F. Zonca, L. Chen, and Y. Wu, Sci. China Earth Sci. 63, 78 (2020).

[70] X. Tao, F. Zonca, and L. Chen, J. Geophys. Res.: Space Phys. 126, e2021JA029585 (2021).

[71] A. Neishtadt and A. Vasiliev, Nonlinearity 18, 1393 (2005).

[72] A. I. Neishtadt, in Hamiltonian Systems with Three or More Degrees of Freedom, edited by C. Simo, NATO ASI Series C, Vol. 533 (Kluwer Academic, Dordrecht, 1999), 193 (1999).

[73] B. V. Chirikov, Sov. J. Plasma Phys. 4, 521 (1978).

[74] A. V. Artemyev, A. I. Neishtadt, and A. A. Vasiliev, Regul. Chaotic Dyn. 25, 2 (2020).
[75] K. G. Orlova and Y. Y. Shprits, Phys. Plasmas 18, 092904 (2011).

[76] A. V. Artemyev, A. I. Neishtadt, A. A. Vasiliev, X.-J. Zhang, D. Mourenas, and D. Vainchtein, J. Plasma Phys. 87, 835870201 (2021).

[77] D. Nunn, X. J. Zhang, D. Mourenas, and A. V. Artemyev, Geophys. Res. Lett. 48, e92178 (2021).

[78] X. J. Zhang, A. G. Demekhov, Y. Katoh, D. Nunn, C. Tao, D. Mourenas, Y. Omura, A. V. Artemyev, and V. Angelopoulos, J. Geophys. Res.: Space Phys. 126, e29330 (2021).

[79] X. Tao, J. Bortnik, R. M. Thorne, J. M. Albert, and W. Li, Geophys. Res. Lett. 39, L06102 (2012).

[80] X. J. Zhang, O. Agapitov, A. V. Artemyev, D. Mourenas, V. Angelopoulos, W. S. Kurth, J. W. Bonnell, and G. B. Hospodarsky, Geophys. Res. Lett. 47, e89807 (2020).

[81] X. J. Zhang, D. Mourenas, A. V. Artemyev, V. Angelopoulos, J. Bortnik, R. M. Thorne, W. S. Kurth, C. A. Kletzing, and G. B. Hospodarsky, Geophys. Res. Lett. 46, 7182 (2019).

[82] Y. Omura, Y. Katoh, and D. Summers, J. Geophys. Res. 113, A04223 (2008).

[83] A. V. Artemyev, D. Mourenas, O. V. Agapitov, and V. V. Krasnoselskikh, Ann. Geophys. 31, 599 (2013).

[84] D. Mourenas, Q. Ma, A. V. Artemyev, and W. Li, Geophys. Res. Lett. 44, 3009 (2017).

[85] S. G. Claudepierre, Q. Ma, J. Bortnik, T. P. O'Brien, J. F. Fennell, and J. B. Blake, Geophys. Res. Lett. 47, e86056 (2020).

[86] O. Allanson, C. E. J. Watt, H. J. Allison, and H. Ratcliffe, J. Geophys. Res.: Space Phys. 126, e28793 (2021).

[87] A. V. Artemyev, D. Mourenas, O. V. Agapitov, D. L. Vainchtein, F. S. Mozer, and V. V. Krasnoselskikh, Phys. Plasmas 22, 082901 (2015). 